\title{
Article \\ Robust Adaptive Path Following Control Strategy for Underactuated Unmanned Surface Vehicles with Model Deviation and Actuator Saturation
}

\author{
Yunsheng Fan ${ }^{1,2, * \mathbb{D}}$, Xinpeng Zou ${ }^{1}$, Guofeng Wang ${ }^{1}$ and Dongdong $\mathrm{Mu}{ }^{1}$ \\ 1 College of Marine Electrical Engineering, Dalian Maritime University, Dalian 116026, China; \\ z1120200191@dlmu.edu.cn (X.Z.); dmuwgf@dlmu.edu.cn (G.W.); ddmu@dlmu.edu.cn (D.M.) \\ 2 Key Laboratory of Technology and System for Intelligent Ships of Liaoning Province, Dalian 116026, China \\ * Correspondence: yunsheng@dlmu.edu.cn; Tel.: +86-135-9134-7650
}

check for updates

Citation: Fan, Y.; Zou, X.; Wang, G.; $\mathrm{Mu}, \mathrm{D}$. Robust Adaptive Path Following Control Strategy for Underactuated Unmanned Surface Vehicles with Model Deviation and Actuator Saturation. Appl. Sci. 2022, 12, 2696. https://doi.org/ 10.3390/app12052696

Academic Editors: Augusto Ferrante and Yosoon Choi

Received: 24 January 2022 Accepted: 24 February 2022 Published: 4 March 2022

Publisher's Note: MDPI stays neutral with regard to jurisdictional claims in published maps and institutional affiliations.

Copyright: () 2022 by the authors. Licensee MDPI, Basel, Switzerland. This article is an open access article distributed under the terms and conditions of the Creative Commons Attribution (CC BY) license (https:// creativecommons.org/licenses/by/ $4.0 /)$.

\begin{abstract}
This paper shows solicitude for the path following control issues of underactuated unmanned surface vehicles subject to unknown external disturbances, deviation of vessel model parameters and actuator saturation. Initially, an improved adaptive integral line-of-sight (IALOS) guidance law is introduced to estimate the sideslip angle, which helps to promote the precision of path following. Furthermore, a finite-time convergent disturbance observer is utilized to size up time-varying disturbances and the single-parameter neural network strategy is utilized to reduce the impact of model deviation. Meanwhile, by introducing a finite-time auxiliary dynamic system to improve the impact of actuator saturation (input saturation), the higher-order tracking differentiator (TDS) is introduced into the backstepping controller for reducing the number of derivations. It is shown that all error signals of the control system, employing Lyapunov stability theory, are uniformly ultimately bounded. Finally, the validity of the put forward scheme is validated by numerical simulations.
\end{abstract}

Keywords: underactuated unmanned surface vehicle; IALOS; path following; model deviation; input saturation; finite-time convergent disturbance observer; TDS

\section{Introduction}

In the last few years, the unmanned surface vehicle (USV) as an intelligent offshore platform facility has appealed to researchers from many fields [1-4] because of its small volume and fast speed. It is widely used in chaotic marine environments, especially when manual intervention is impossible.

The path following control of the USV drives the vehicle to accurately track the expected route without strict time constraints [5-7]. Nowadays, line-of-sight (LOS) guidance, which provides path information for a path following controller, is a main research direction of USV path following control as a result of its high efficiency and simplicity. Paper [8] verifies that the proportional LOS guidance method is simple and practical. Papers [9-12] improve the LOS guidance method by introducing the integral term, which effectively improves the influence under the condition of constant flow. For the sake of handling the issue of underactuated vehicle path following with uncertainty and input saturation, an improved LOS (ILOS) guidance method is proposed in Paper [13]. A predictor-based LOS (PLOS) guidance method, which effectively reduces the influence of sideslip angle and obtains a more accurate guidance control law, is proposed in Paper [14]. Based on an extended state observer (ESO), Paper [15] proposes an ESO LOS (ELOS) guidance method to handle the issue of time-varying sideslip angle and to improve the path following effect of underactuated unmanned vehicles when drift angle changes. Paper [16] puts forward an IALOS guidance law to effectively improve the path following effect of USVs when sideslip angle and ocean current are time-varying. Paper [17] proposes ocean current 
observer LOS guidance, which can more quickly compensate the impact of ocean current on path following.

Paper [18] proposes a compound LOS (CLOS) guidance law to solve the problem of excessive sideslip angle. For the sake of overcoming the issue of time delay caused by stochastic differential rules, a global robust adaptive controller is designed in Paper [19] by drawing into weak and strong nonlinear Lyapunov functions. Sliding mode control (SMC) is extensively utilized in the field of vehicle control on account of its strong robustness and fast response. The integral sliding mode control method is applied to unmanned surface vehicles for the first time in Paper [20]. Paper [21] designs a fast nonsingular terminal $\mathrm{SMC}$, which has the advantage of rapidity and robustness. Papers [22,23] design auxiliary dynamic systems to approach the actuator saturation issue in the course of vehicle motion in practice. In [24], based on the finite time theory, an external time-varying disturbance is quickly approached in a finite time by putting forward a finite-time disturbance observer (FCDO). Paper [25], based on minimal learning parameter (MLP) technology and neural network technology, proposes a neural network disturbance observer to effectively solve the observation of disturbance when the vehicle model is uncertain. Paper [26] proposes a robust adaptive neural track tracking control strategy by combining dynamic surface control (DSC) with the MLP method. The designed adaptive law replaces the estimation of neural network weight itself by estimating the norm of neural network weight online, which effectively solves the problem of excessive computation caused by adaptive neural networks in Paper [27,28]. For the sake of overcoming the issue that the parameter learning time of neural network technology is too long, resulting in poor real-time performance and difficult implementation in practical engineering, a single-parameter neural network is proposed in Paper [29]. Papers [30-34] employ model-predictive-control methods, efficiently settling the issue of model uncertainty.

Enlightened by the aforesaid observations, IALOS guidance is introduced to supply guidance. Moreover, a path following control policy is designed by applying a singleparameter neural network, a finite-time auxiliary dynamic system, a higher-order tracking differentiator and a finite-time disturbance observer. The main characteristics of the proposed control scheme are summarized as follows:

1 Considering the practical application requirements of path following control, some practical problems encountered in the process of vessel motion are taken into consideration, For instance, these problems include unknown external time-varying disturbances, deviation of vehicle model parameters and actuator saturation. The above problems are solved by disturbance observers, neural networks and auxiliary dynamic systems, respectively.

2 For the sake of reducing the complexity of the control policy, the following two measures are taken in this paper. (1) The higher-order tracking differentiator (TDS) is introduced into the backstepping controller, which reduces the number of derivations in the backstepping controller and overcomes the issue of complex calculation of the controller. (2) The single-parameter approximation strategy is used to approximate the vehicle model. Because the single-parameter neural network only needs to adjust one parameter online, the complexity and amount of calculation of the controller are effectively reduced compared with Paper [16].

3 The rapidity of the control system is taken into account. (1) FCDO is devised to approach the external time-varying disturbance, which effectively speeds up the convergence speed of the proposed scheme. (2) A finite-time auxiliary dynamic system is adopted for the actuator saturation issue to further accelerate the convergence speed of the control system compared with Paper [17].

The rest of this paper is organized as follows: Problem formulation is put forward in Section 2. In Section 3, IALOS guidance law is utilized. In Section 4, the path following policy is presented. In Section 5, analysis of system stability is formulated. In Section 6, simulation results are presented to testify of the excellence of the control strategy. The full text is concluded in Section 7. 


\section{Problem Formulation}

$O-X_{0} Y_{0} Z_{0}$, which denotes the Earth-fixed inertial frame, and $X Y Z$, which denotes the body-fixed frame, are revealed in Figure 1.

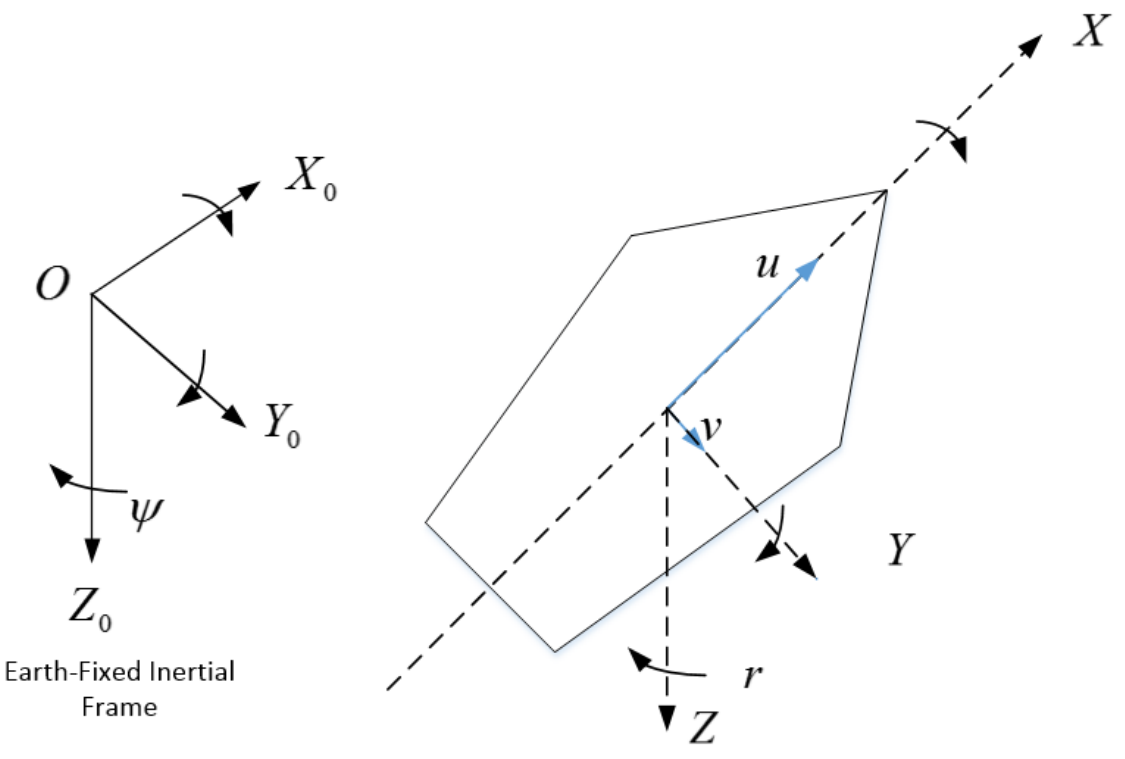

Body-Fixed Frame

Figure 1. The Earth-fixed inertial and body-fixed frames.

The kinematics equation is indicated by

$$
\dot{\zeta}=J(\psi) v
$$

where $\zeta=[x, y, \psi]^{T}$ denotes the position and the heading angle of the USV, respectively. $v=[u, v, r]^{T}$ denotes surge velocity, sway velocity and yaw rate, respectively. $J(\psi)$ represents the transition matrix:

$$
J(\psi)=\left[\begin{array}{ccc}
\cos (\psi) & -\sin (\psi) & 0 \\
\sin (\psi) & \cos (\psi) & 0 \\
0 & 0 & 1
\end{array}\right]
$$

Then, the kinematic equations can be expressed as [35].

$$
\left\{\begin{array}{l}
\dot{x}=u \cos \psi-v \sin \psi \\
\dot{y}=u \sin \psi+v \cos \psi \\
\dot{\psi}=r
\end{array}\right.
$$

According to Paper [13], the dynamic equations of an underactuated USV model can be represented by

$$
\left\{\begin{array}{l}
\dot{u}=\frac{m_{22}}{m_{11}} v r-\frac{d_{11}}{m_{11}} u+\frac{\tau_{u}+d_{u}}{m_{11}} \\
\dot{v}=-\frac{m_{11}}{m_{22}} u r-\frac{d_{22}}{m_{22}} v+\frac{d_{v}}{m_{22}} \\
\dot{r}=\frac{m_{11}-m_{22}}{m_{33}} u v-\frac{d_{33}}{m_{33}} r+\frac{\tau_{r}+d_{r}}{m_{11}}
\end{array}\right.
$$

where $\tau_{u}$ and $\tau_{r}$ denote the control input of surge force and the control input of yaw moment, respectively. $b_{u}, b_{v}$ and $b_{r}$ represent immeasurable external environmental disturbances owing to waves, wind and ocean current. 
Define

$$
\left\{\begin{array}{l}
f_{u}=\frac{m_{22}}{m_{11}} v r-\frac{d_{11}}{m_{11}} u \\
f_{v}=-\frac{m_{11}}{m_{22}} u r-\frac{d_{22}}{m_{22}} v \\
f_{r}=\frac{m_{11}-m_{22}}{m_{33}} u v-\frac{d_{33}}{m_{33}} r
\end{array}\right.
$$

In practice, the control inputs have certain boundaries. They can be be represented by

$$
\tau_{i c}=\left\{\begin{array}{l}
\tau_{i \max }, \quad \text { if } \tau_{i}>\tau_{i \max } \\
\tau_{i}, \quad \text { if } \tau_{i \min } \leq \tau_{i} \leq \tau_{i \max } \\
\tau_{i \min }, \quad \text { if } \tau_{i}<\tau_{i \min }
\end{array}\right.
$$

where $i=u, r$ and $\tau_{i \max }$ and $\tau_{i \min }$ denote upper and lower limits of actuator input, respectively. $\tau_{i}$ denotes the input command without input saturation and $\tau_{i c}$ denotes the ultimate input command.

Remark 1. In essence, $f_{u}, f_{v}$ and $f_{r}$ are biased. In practice, the ship model should be $\bar{f}_{u}=f_{u}+\Delta f_{u}$, $\bar{f}_{v}=f_{v}+\Delta f_{v}$ and $\bar{f}_{r}=f_{r}+\Delta f_{r}$.

Remark 2. It has been proved in [36] that the sway $v$ is passive-bounded stable.

Assumption 1. The unknown disturbance is bounded. $\left|b_{u}\right| \leq b_{u \max },\left|b_{v}\right| \leq b_{v \max }$ and $\left|b_{r}\right| \leq b_{r \max }$, where $b_{u \max }, b_{v \max }$ and $b_{r \max }$ denote maximum disturbance, respectively.

Assumption 2. Control inputs and velocities are bounded. $\tau_{u \min } \leq \tau_{u} \leq \tau_{u \max }$ $\tau_{r \min } \leq \tau_{r} \leq \tau_{r \max }, u \leq u_{\max }, v \leq v_{\max }$ and $r \leq r_{\max }$ with positive constants $\tau_{u \max }$ $\tau_{r \max }, u_{\max }, v_{\max }$ and $r_{\max }$ and negative constants $\tau_{u \min }$ and $\tau_{r \min }$.

\section{LOS Guidance Algorithms}

A geometric path $\left(x_{p}(\theta), y_{p}(\theta)\right)$ is considered the reference path, where $\theta$ denotes an independent variable. The path-tangential reference frame of the along-track error $x_{e}$ and the cross-track error $y_{e}$ is computed as follows:

$$
\left[\begin{array}{l}
x_{e} \\
y_{e}
\end{array}\right]=R^{T}\left(\gamma_{p}\right)\left[\begin{array}{l}
x-x_{p}(\theta) \\
y-y_{p}(\theta)
\end{array}\right]
$$

where

$$
R\left(\gamma_{p}\right)=\left[\begin{array}{cc}
\cos \left(\gamma_{p}\right) & -\sin \left(\gamma_{p}\right) \\
\sin \left(\gamma_{p}\right) & \cos \left(\gamma_{p}\right)
\end{array}\right]
$$

where $R\left(\gamma_{p}\right) \in S O(2)$ denotes the rotation matrix in yaw and

$$
\gamma_{p}(\theta)=\arctan \left(y_{p}^{\prime}(\theta), x_{p}^{\prime}(\theta)\right)
$$

where $x_{p}^{\prime}(\theta)=\partial x_{p} / \partial_{\theta}$ and $y_{p}^{\prime}(\theta)=\partial y_{p} / \partial_{\theta}$.

Based on Equation (8), it is found that

$$
\left\{\begin{array}{l}
x_{e}=\left(x-x_{p}(\theta)\right) \cos \left(\gamma_{p}\right)+\left(y-y_{p}(\theta)\right) \sin \left(\gamma_{p}\right) \\
y_{e}=-\left(x-x_{p}(\theta)\right) \sin \left(\gamma_{p}\right)+\left(y-y_{p}(\theta)\right) \cos \left(\gamma_{p}\right)
\end{array}\right.
$$

Differentiating $x_{e}$ and $y_{e}$ along (12) and (13) gives:

$$
\begin{gathered}
\dot{x}_{e}=U \cos \left(\psi-\gamma_{p}+\beta\right)+\dot{\gamma}_{p} y_{e}-U_{p} \\
\dot{y}_{e}=U \sin \left(\psi-\gamma_{p}+\beta\right)+\dot{\gamma}_{p} y_{e}
\end{gathered}
$$


where $U=\sqrt{u^{2}+v^{2}}, \beta=\arctan (v, u)$ represents the sideslip angle and $U_{p p}=\dot{\theta} \sqrt{x_{p}^{\prime 2}+y_{p}^{\prime 2}}$ denotes the virtual reference speed to stabilize $x_{e}$. A local path parallel reference frame is revealed in Figure 2.

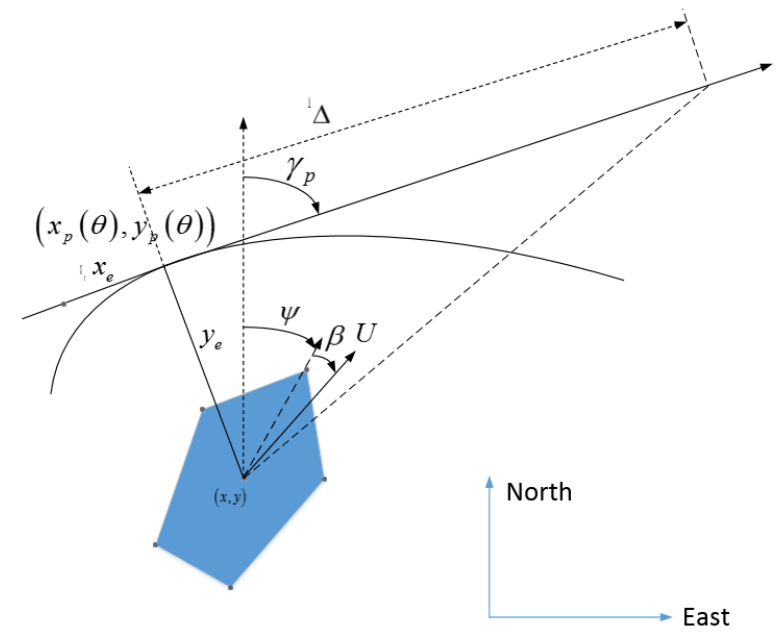

Figure 2. LOS guidance geometry for curved paths.

Assumption 3. During unmanned surface vessel path following, $\beta$ is relatively small, $|\beta| \leq \beta^{*}$ and $\beta^{*}$ denotes a positive constant number. It is assumed that $\beta$ changes slowly. $|\dot{\beta}| \leq C_{\beta}$ and $C_{\beta}$ denotes a positive constant number. Therefore, $\sin (\beta)=\beta$ and $\cos (\beta)=1$.

Rewrite the position error derivatives as the following:

$$
\begin{gathered}
\dot{x}_{e}=U \cos \left(\psi-\gamma_{p}\right)-U \sin \left(\psi-\gamma_{p}\right) \beta-U_{p p}+y_{e} \dot{\gamma}_{p} \\
\dot{y}_{e}=U \sin \left(\psi-\gamma_{p}\right)+U \cos \left(\psi-\gamma_{p}\right) \beta
\end{gathered}
$$

Define $\hat{\beta}$ as an estimated value of $\beta . \tilde{\beta}=\beta-\hat{\beta}$ denotes the estimation error. Design the Lyapunov function as follows:

$$
V_{g}=\frac{1}{2} x_{e}^{2}+\frac{1}{2} y_{e}^{2}+\frac{1}{2 k_{\beta}} \tilde{\beta}^{2}
$$

where $k_{\beta}$ is the positive design parameters.

Take the time derivative Equation (16) along (14) and (15); then, the following equation is obtained:

$$
\begin{aligned}
\dot{V}_{g}= & x_{e}\left[U \cos \left(\psi-\gamma_{p}\right)-U \sin \left(\psi-\gamma_{p}\right) \beta+\dot{\gamma}_{p} y_{e}-U_{p p}\right] \\
& +y_{e}\left[U \sin \left(\psi-\gamma_{p}\right)+U \cos \left(\psi-\gamma_{p}\right) \beta-\dot{\gamma}_{p} x_{e}\right]+\frac{1}{k_{\beta}} \tilde{\beta}(\dot{\beta}-\dot{\hat{\beta}})
\end{aligned}
$$

IALOS can effectively compute the anticipated yaw angle. In practice, $\beta$ is often difficult to measure, so the adaptive strategy is employed to estimate the time-varying $\beta$.

Assumption 4. The reference heading angle can followed perfectly; therefore, $\psi=\psi_{d}$.

Based on (15), the desired heading angle, the sideslip angle adaptive law and the path parameter update law are obtained [16].

$$
\left.\psi_{d}=\gamma_{p}-\operatorname{acr} \tan \left(\frac{1}{\Delta} y_{e}+\hat{\beta}\right)\right)
$$




$$
\dot{\hat{\beta}}=k_{\beta}\left[\frac{U y_{e} \Delta}{\sqrt{\Delta^{2}+\left(y_{e}+\Delta \hat{\beta}\right)^{2}}}-\hat{\beta}\right]
$$

and

$$
\dot{\theta}=\frac{U \cos \left(\psi-\gamma_{p}\right)+k_{s} x_{e}}{\sqrt{x^{\prime}{ }_{p}(\theta)^{2}+y_{p}^{\prime}(\theta)^{2}}}
$$

where $\Delta$ denotes the positive design parameters.

Based on Equation (18), it is found that

$$
\begin{aligned}
& \sin \left(\psi-\gamma_{p}\right)=-\frac{y_{e}+\Delta \hat{\beta}}{\sqrt{\Delta^{2}+\left(y_{e}+\Delta \hat{\beta}\right)^{2}}} \cos \psi_{e}+\frac{\Delta}{\sqrt{\Delta^{2}+\left(y_{e}+\Delta \hat{\beta}\right)^{2}}} \sin \psi_{e} \\
& \cos \left(\psi-\gamma_{p}\right)=\frac{\Delta}{\sqrt{\Delta^{2}+\left(y_{e}+\Delta \hat{\beta}\right)^{2}}} \cos \psi_{e}+\frac{y_{e}+\Delta \hat{\beta}}{\sqrt{\Delta^{2}+\left(y_{e}+\Delta \hat{\beta}\right)^{2}}} \sin \psi_{e}
\end{aligned}
$$

where $\psi_{e}=\psi-\psi_{d}$. Substituting (18)-(20) into (17),

$$
\begin{aligned}
\dot{V}_{g}= & -k_{s} x_{e}^{2}-U x_{e} \sin \left(\psi-\gamma_{p}\right) \beta-\frac{y_{e} U\left(y_{e}-\Delta \tilde{\beta}\right)}{\sqrt{\Delta^{2}+\left(y_{e}+\Delta \hat{\beta}\right)^{2}}}+y_{e} U\left(\frac{\Delta+y_{e} \beta+\Delta \hat{\beta} \beta}{\sqrt{\Delta^{2}+\left(y_{e}+\Delta \hat{\beta}\right)^{2}}}\right) \sin \psi_{e} \\
& +y_{e} U\left(1-\cos \psi_{e}\right) \frac{y_{e}-\Delta \tilde{\beta}}{\sqrt{\Delta^{2}+\left(y_{e}+\Delta \hat{\beta}\right)^{2}}}+\frac{1}{k_{\beta}} \tilde{\beta} \dot{\beta}-\tilde{\beta}\left[\frac{y_{e} U \Delta}{\sqrt{\left(y_{e}+\Delta \hat{\beta}\right)^{2}+\Delta^{2}}}-\hat{\beta}\right]
\end{aligned}
$$

Based on Young's inequality, the following equation is obtained:

$$
-U x_{e} \sin \left(\psi-\gamma_{p}\right) \beta \leq \frac{1}{2} U x_{e}^{2}+\frac{1}{2} U \beta^{* 2}
$$

Substituting (24) into (23),

$$
\dot{V}_{\mathrm{g}} \leq-\left(k_{s}-\frac{1}{2} U\right) x_{e}^{2}-\frac{U}{\sqrt{\Delta^{2}+\left(y_{e}+\Delta \hat{\beta}\right)^{2}}} y_{e}^{2}-\tilde{\beta}^{2}+y_{e} U \phi \psi_{e}+|\tilde{\beta}|\left(\frac{C_{\beta}}{k_{\beta}}+\beta^{*}\right)+\frac{1}{2} U \beta^{* 2}
$$

where $\delta=\frac{\left(1-\cos \psi_{e}\right)\left(y_{e}-\Delta \tilde{\beta}\right)+\left(\Delta+y_{e} \beta+\Delta \hat{\beta} \beta\right) \sin \psi_{e}}{\psi_{e} \sqrt{\Delta^{2}+\left(y_{e}+\Delta \hat{\beta}\right)^{2}}}$.

$$
\begin{aligned}
& \text { Owing to }\left|\frac{\sin \psi_{e}}{\psi_{e}}\right| \leq 1,\left|\frac{1-\cos \psi_{e}}{\psi_{e}}\right|<0.73,\left|\frac{y_{e}+\Delta \hat{\beta}}{\sqrt{\Delta^{2}+\left(y_{e}+\Delta \hat{\beta}\right)^{2}}}\right| \leq 1 \text { and }\left|\frac{\Delta}{\sqrt{\Delta^{2}+\left(y_{e}+\Delta \hat{\beta}\right)^{2}}}\right| \leq 1, \\
& \begin{aligned}
\delta= & \frac{\left(1-\cos \psi_{e}\right)}{\psi_{e}}\left(\frac{y_{e}+\Delta \hat{\beta}}{\sqrt{\Delta^{2}+\left(y_{e}+\Delta \hat{\beta}\right)^{2}}}-\frac{\Delta \beta}{\sqrt{\Delta^{2}+\left(y_{e}+\Delta \hat{\beta}\right)^{2}}}\right) \\
& +\frac{\sin \psi_{e}}{\psi_{e}}\left(\frac{\Delta}{\sqrt{\Delta^{2}+\left(y_{e}+\Delta \hat{\beta}\right)^{2}}}+\frac{\left(y_{e}+\Delta \hat{\beta}\right) \beta}{\sqrt{\Delta^{2}+\left(y_{e}+\Delta \hat{\beta}\right)^{2}}}\right) \leq 1.73+1.73 \beta^{*}
\end{aligned}
\end{aligned}
$$

\section{Control System Design}

Figure 3 reveals the block diagram of the proposed control strategy. 


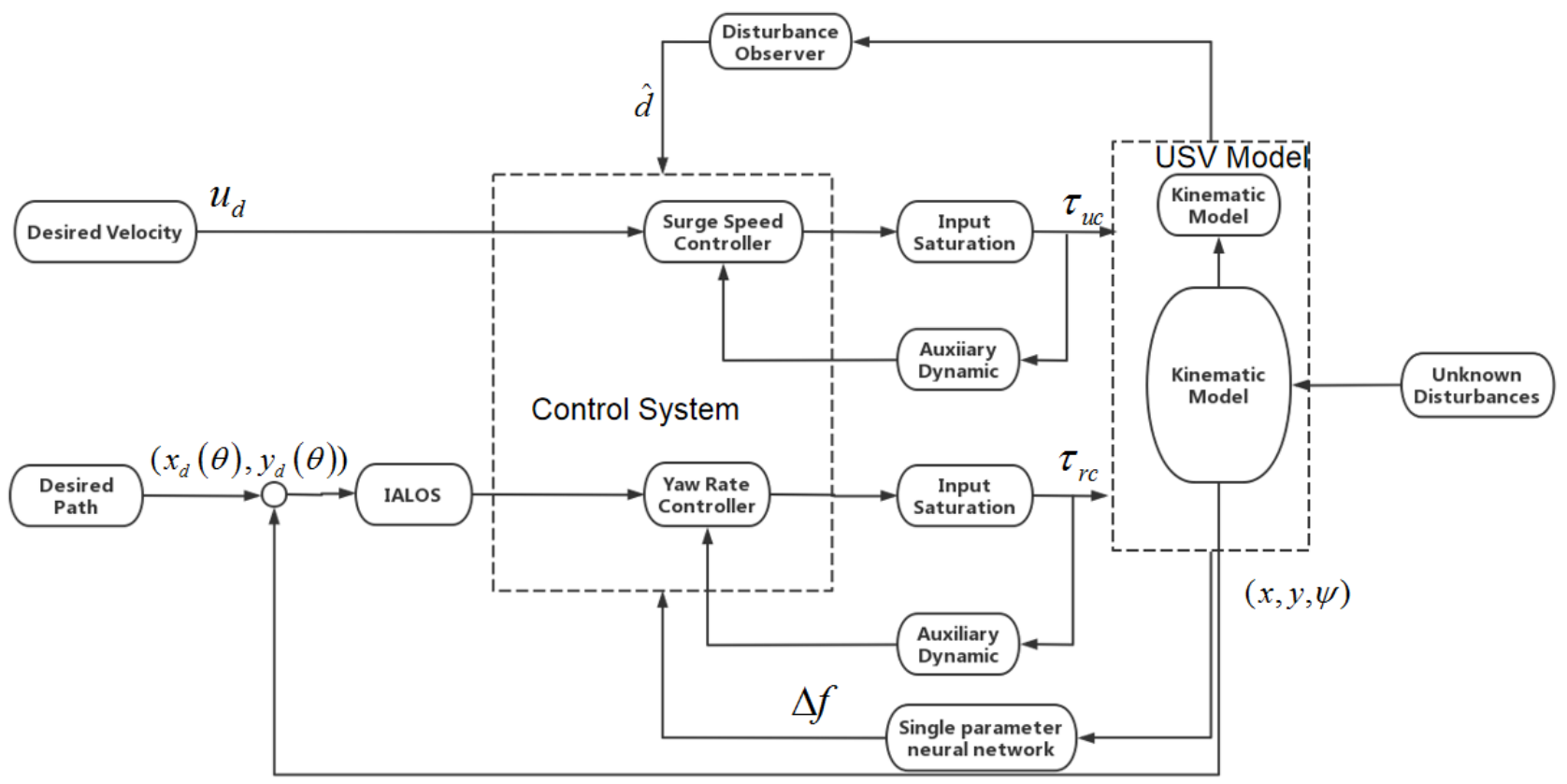

Figure 3. The block diagram of USV path following.

\subsection{Finite-Time Convergent Disturbance Observer Design}

For the sake of promoting the design of FCDO [24], facilitate (4) as follows:

$$
M \dot{v}+C v+D v=\tau+d
$$

Firstly, define accessory variable $p=M v$, where $\hat{p}$ denotes the estimate of $p$. Furthermore, $\tilde{p}$ denotes the estimation error of accessory variable $p$ :

$$
\tilde{p}=\hat{p}-p
$$

Differentiating Equation (28),

$$
\begin{aligned}
\dot{\hat{p}}= & -C v-D v+\tau-D_{1} \tilde{p}-D_{2}|\tilde{p}|^{\gamma} \operatorname{sign}(\tilde{p}) \\
& -\bar{d} \operatorname{sign}(\tilde{p})
\end{aligned}
$$

where $D_{1}=\operatorname{diag}\left\{D_{11}, D_{12}, D_{13}\right\}$ and $D_{2}=\operatorname{diag}\left\{D_{21}, D_{22}, D_{23}\right\}$ represent FCDO parameter matrices; $D_{1 i}$ and $D_{2 i}(i=1,2,3)$ denote positive design parameters; $\bar{d}=\left[\bar{d}_{u}, \bar{d}_{v}, \bar{d}_{r}\right]$ denotes upper bound vector of disturbance; $0<\gamma<1$; $\hat{d}$ denotes the estimate of $d$ and $\hat{d}$ can be further acquired by (29):

$$
\begin{aligned}
& \hat{d}=\dot{\hat{p}}+C v+D v-\tau \\
& =-D_{1} \tilde{p}-D_{2}|\tilde{p}|^{\gamma} \operatorname{sign}(\tilde{p})-\bar{d} \operatorname{sign}(\tilde{p})
\end{aligned}
$$

Consider the following Lyapunov function candidate:

$$
V_{p}=\frac{1}{2} \tilde{p}^{T} \tilde{p}
$$

Differentiating Equation (31),

$$
\dot{V}_{p}=\tilde{p}^{T} \dot{\tilde{p}}=\tilde{p}^{T}(\dot{p}-M \dot{v})
$$


Substituting (27) and (29) into (32) results in

$$
\begin{aligned}
\dot{V}_{p} & =\tilde{p}^{T}\left(-D_{1} \tilde{p}-D_{2}|\tilde{p}|^{\gamma} \operatorname{sign}(\tilde{p})-\bar{d} \operatorname{sign}(\tilde{p})-d\right) \\
& \leq-\tilde{p}^{T} D_{1} \tilde{p}-\tilde{p}^{T} D_{2}|\tilde{p}|^{\gamma} \operatorname{sign}(\tilde{p}) \\
& \leq-2 D_{1 \min } V_{p}-2^{\gamma+1} D_{2 \min } V_{p}^{(\gamma+1) / 2}
\end{aligned}
$$

where $D_{1 \min }=\min \left\{D_{11}, D_{12}, D_{13}\right\}$ and $D_{2 \min }=\min \left\{D_{21}, D_{22}, D_{23}\right\}$.

According to Paper [37], $\tilde{p}$ converges to 0. Further acquired from (28)-(30) is the following:

$$
\begin{aligned}
\tilde{d}= & d-\hat{d} \\
= & D_{1} \tilde{p}+D_{2}|\tilde{p}|^{\gamma} \operatorname{sign}(\tilde{p})+\bar{d} \operatorname{sign}(\tilde{p}) \\
& \quad+M \dot{v}+C v+D v-\tau \\
= & \dot{p}-\dot{\hat{p}}=-\dot{\tilde{p}}
\end{aligned}
$$

\subsection{Yaw Rate Controller}

The heading tracking error $\psi_{e}$ is defined as follows:

$$
\psi_{e}=\psi-\psi_{d}
$$

Hence, $\dot{\psi}_{e}=r-\dot{\psi}_{d}$.

Consider the following Lyapunov function candidate:

$$
V_{\psi}=\frac{1}{2} \tilde{\psi}^{2}
$$

Differentiating Equation (36),

$$
\dot{V}_{\psi}=\tilde{\psi}\left(r-\dot{\psi}_{d}\right)
$$

Define the yaw angle rate tracking error variable

$$
\tilde{r}=r-r_{d}
$$

where $r_{d}$ denotes virtual control input:

$$
r_{d}=-k_{\psi} \tilde{\psi}+\dot{\psi}_{d}-U \delta y_{e}
$$

where $k_{\psi}$ is a positive control design parameter.

Next,

$$
V_{\psi}=-k_{\psi} \tilde{\psi}^{2}+\tilde{\psi} \tilde{r}-U \phi y_{e} \tilde{\psi}
$$

According to Remark 1 and Equation (4), differentiating Equation (38),

$$
\dot{\tilde{r}}=\dot{r}-\dot{r}_{d}=\bar{f}_{r}+\frac{\tau_{r}}{m_{33}}+\frac{d_{r}}{m_{33}}-\dot{r}_{d}
$$

Consider the following Lyapunov function:

$$
V_{r}=V_{\psi}+\frac{1}{2} m_{33} \tilde{r}^{2}
$$

Differentiating Equation (42),

$$
\begin{aligned}
\dot{V}_{r} & =\dot{V}_{\psi}+m_{33} \tilde{r} \dot{\tilde{r}} \\
& =-k_{\psi} \tilde{\psi}^{2}+\tilde{\psi} \tilde{r}-U \phi y_{e} \tilde{\psi}+\tilde{r}\left(m_{33} \bar{f}_{r}+\tau_{r}+d_{r}-m_{33} \dot{r}_{d}\right)
\end{aligned}
$$


Based on Equation (43), the design heading path following control law is as follows:

$$
\tau_{r}=-k_{r} \tilde{r}+m_{33} \dot{r}_{d}-m_{33} \bar{f}_{r}-d_{r}-\tilde{\psi}
$$

where $k_{r}>0$ denotes control design parameters.

Since the model has deviation in practice, the model deviation is approximated by utilizing the single-parameter neural network [29].

$$
\left|\Delta f_{r}\right|=\left|\frac{k_{r \Delta}\left(m_{11}-m_{22}\right)}{m_{33}} u v-\frac{k_{r \Delta} d_{33}}{m_{33}} r\right| \leq \Theta_{r} \hat{\phi}(r)
$$

where

$$
\hat{\phi}(r)=|u v|+|r|
$$

$\Theta_{r}$ has no clear physical meaning, so it is called a virtual parameter.

$$
\Theta_{r}=\max \left\{\left|\frac{k_{r \Delta}\left(m_{11}-m_{22}\right)}{m_{33}}\right|,\left|\frac{k_{r \Delta} d_{33}}{m_{33}}\right|\right\}
$$

The third-order TD with $\psi_{d}$ current as input signal is designed as follows:

$$
\left\{\begin{array}{l}
\dot{v}_{1}=v_{2} \\
\dot{v}_{2}=v_{3} \\
\dot{v}_{3}=-l_{1}^{3}\left(a_{1}\left(v_{1}-\psi_{d}\right)+a_{2} v_{2} / \iota_{1}+a_{3} v_{3} / \iota_{1}^{2}\right)
\end{array}\right.
$$

where $\iota_{1}, a_{1}, a_{2}$ and $a_{3}$ are positive constants; $v_{1}, v_{2}$ and $v_{3}$ denote the corresponding estimations, which are the state variables of TD; $v_{1}=\psi_{d c} ; v_{2}=\dot{\psi}_{d c}$ and $v_{3}=\ddot{\psi}_{d c}$.

Define $\vartheta=U \delta y_{e}$, the second-order TD with $\vartheta$ current as input signal, is designed as follows:

$$
\left\{\begin{array}{l}
v_{4}=-v_{5} \\
v_{5}=-l_{2}^{2}\left(a_{4}\left(v_{4}-\vartheta\right)+a_{5} v_{5} / \iota_{2}\right)
\end{array}\right.
$$

where $\iota_{2}, a_{4}$ and $a_{5}$ denote positive design parameters; $v_{4}$ and $v_{5}$ denote the corresponding estimates, which are the state variables of TD; $v_{4}=\vartheta_{c}$ and $v_{5}=\dot{\vartheta}_{c}$.

Substituting (48) and (49) into (39), the following equation is obtained:

$$
\dot{r}_{d}=-k_{2}\left(r-\dot{\psi}_{d c}\right)+\ddot{\psi}_{d c}-\dot{\vartheta}_{c}
$$

In light of Formula (44), the adaptive single-parameter neural network yaw rate controller can be proposed as

$$
\tau_{r}=-k_{r} \tilde{r}-m_{33} k_{\psi}\left(r-\dot{\psi}_{d c}\right)+m_{33} \ddot{\psi}_{d c}-m_{33} \dot{\vartheta}_{c}-\hat{d}_{r}-m_{33} \bar{f}_{r}-m_{33} c_{r} \hat{\Theta}_{r} \hat{\phi}^{2}(r) \tilde{r}-\tilde{\psi}
$$

with the adaptive law

$$
\dot{\hat{\Theta}}_{r}=c_{r} \hat{\phi}^{2}(r) \tilde{r}^{2}-\sigma_{r} \hat{\Theta}_{r}, \quad \hat{\Theta}_{r}(0) \geq 0
$$

For the sake of reducing the impact of actuator saturation on the control system [38], the following auxiliary dynamic system equation is constructed:

$$
\dot{\zeta}_{r}=-k_{\zeta r 1} \zeta_{r}-k_{\zeta r 0} S_{\zeta_{r}}+\Delta \tau_{r}
$$

where

$$
S_{\zeta_{r}}= \begin{cases}\left|\zeta_{r}\right|^{\frac{1}{2}} \operatorname{sgn}\left(\zeta_{r}\right) & \left|\zeta_{r}\right| \geq \varepsilon_{r} \\ \frac{3}{2} \varepsilon_{r}^{-\frac{1}{2}} \zeta_{r}-\frac{1}{2} \varepsilon_{r}^{-\frac{3}{2}} \zeta_{r}^{2} & \left|\zeta_{r}\right|<\varepsilon_{r}\end{cases}
$$


The designed control law for the yaw angle subsystem is $\tau_{r c}=-k_{r} \tilde{r}-m_{33} k_{\psi}\left(r-\dot{\psi}_{d c}\right)+m_{33} \ddot{\psi}_{d c}-m_{33} \dot{\theta}_{c}-\hat{d}_{r}-m_{33} f_{r}-m_{33} c_{r} \hat{\Theta}_{r} \hat{\phi}^{2}(r) \tilde{r}-\tilde{\psi}+m_{33} k_{\tau_{r}} \zeta_{r}$

\subsection{Surge Speed Controller}

The velocity tracking error $\tilde{u}$ is defined as follows:

$$
\tilde{u}=u-u_{d}
$$

According to Remark 1 and Equation (4), differentiating Equation (56) gives

$$
\dot{\tilde{u}}=\bar{f}_{u}+\frac{\tau_{u}}{m_{11}}+\frac{d_{u}}{m_{11}}-\dot{u}_{d}
$$

Consider the following Lyapunov function:

$$
V_{u}=\frac{1}{2} m_{11} \tilde{u}^{2}
$$

Differentiating Equation (58),

$$
\begin{aligned}
& \dot{V}_{u}=m_{11} \tilde{u} \dot{\tilde{u}} \\
& \quad=\tilde{u}\left(m_{11} \bar{f}_{u}+\tau_{u}+d_{u}-m_{11} \dot{u}_{d}\right)
\end{aligned}
$$

The corresponding control law is chosen as

$$
\tau_{u}=-k_{u} \tilde{u}-m_{11} \bar{f}_{u}-d_{u}+m_{11} \dot{u}_{d}
$$

Since the model has deviation in practice, the model deviation is approximated by utilizing the single-parameter neural network

$$
\left|\Delta f_{u}\right|=\left|\frac{k_{u \Delta} m_{22}}{m_{11}} v r-\frac{k_{u \Delta} d_{11}}{m_{11}} u\right| \leq \Theta_{u} \hat{\phi}(u)
$$

where

$$
\hat{\phi}(u)=|v r|+|u|
$$

$\Theta_{u}$ has no clear physical meaning, so it is called a virtual parameter.

$$
\Theta_{u}=\max \left\{\left|\frac{k_{u \Delta} m_{22}}{m_{11}}\right|,\left|\frac{k_{u \Delta} d_{11}}{m_{11}}\right|\right\}
$$

In light of Formula (60), the adaptive single-parameter neural network surge speed controller can be proposed as

$$
\tau_{u}=-k_{u} \tilde{u}-m_{11} f_{u}-\hat{d}_{u}+m_{11} \dot{u}_{d}-m_{11} c_{u} \hat{\Theta}_{u} \hat{\phi}^{2}(u) \tilde{u}
$$

with the adaptive law

$$
\dot{\hat{\Theta}}_{u}=c_{u} \hat{\phi}^{2}(u) \tilde{r}^{2}-\sigma_{u} \hat{\Theta}_{u}, \quad \hat{\Theta}_{u}(0) \geq 0
$$

For the sake of reducing the impact of actuator saturation on the control system, the following auxiliary dynamic system equation is established:

$$
\dot{\zeta}_{u}=-k_{\zeta u 1} \zeta_{u}-k_{\zeta u 0} S_{\zeta u}+\Delta \tau_{u}
$$


where

$$
S_{\zeta_{u}}= \begin{cases}\left|\zeta_{u}\right|^{\frac{1}{2}} \operatorname{sgn}\left(\zeta_{u}\right) & \left|\zeta_{u}\right| \geq \varepsilon_{u} \\ \frac{3}{2} \varepsilon_{u}^{-\frac{1}{2}} \zeta_{u}-\frac{1}{2} \varepsilon_{u}^{-\frac{3}{2}} \zeta_{u}^{2} & \left|\zeta_{u}\right|<\varepsilon_{u}\end{cases}
$$

The designed control law for the surge speed subsystem is

$$
\tau_{u c}=-k_{u} \tilde{u}-m_{11} f_{u}-\hat{d}_{u}+m_{11} \dot{u}_{d}-m_{11} c_{u} \hat{\Theta}_{u} \hat{\phi}^{2}(u) \tilde{u}+m_{11} k_{\zeta u} \zeta_{u}
$$

\section{Stability Analysis}

Define

$$
V_{r a}=V_{\psi}+\frac{m_{33} \tilde{r}^{2}}{2}+\frac{m_{33} \tilde{\Theta}_{r}^{2}}{2}+\frac{m_{33} \zeta_{r}^{2}}{2}
$$

Differentiating Equation (69),

$$
\begin{aligned}
\dot{V}_{r a} & =\tilde{\psi} \dot{\tilde{\psi}}+m_{33} \tilde{r} \tilde{r}-m_{33} \tilde{\Theta}_{r} \dot{\hat{\Theta}}_{r}+\mathrm{m}_{33} \zeta_{r} \dot{\zeta}_{r} \\
& =-k_{\psi} \tilde{\psi}^{2}-U \phi y_{e} \tilde{\psi}+\tilde{r}\left(m_{33} \Delta f_{r}-k_{r} \tilde{r}-m_{33} c_{r} \hat{\Theta}_{r} \hat{\phi}^{2}(r) \tilde{r}+m_{33} k_{\zeta r} \zeta_{r}+\tilde{d}_{r}\right) \\
& -m_{33} c_{r} \hat{\phi}^{2}(r) \tilde{\Theta}_{r} \tilde{r}^{2}+m_{33} \sigma_{r} \hat{\Theta}_{r} \tilde{\Theta}_{r}+m_{33}\left(-k_{\zeta r 1} \zeta_{r}^{2}-k_{\zeta r 0}\left|\zeta_{r}\right|^{\frac{3}{2}}+\zeta_{r} \Delta \tau_{r}\right)
\end{aligned}
$$

Substituting (45) into (69), it is obtained that

$$
\begin{aligned}
\dot{V}_{r a} \leq & -k_{\psi} \tilde{\psi}^{2}-U \phi y_{e} \tilde{\psi}-k_{r} \tilde{r}^{2}+\tilde{r}\left(m_{33} \hat{\Theta}_{r} \hat{\phi}(r)-m_{33} c_{r} \hat{\Theta}_{r} \hat{\phi}^{2}(r) \tilde{r}+m_{33} k_{\zeta r} \zeta_{r}+\Delta \tau_{r}+\tilde{d}_{r}\right) \\
& -m_{33} c_{r} \hat{\phi}^{2}(r) \tilde{\Theta}_{r} \tilde{r}^{2}+m_{33} \sigma_{r} \hat{\Theta}_{r} \tilde{\Theta}_{r}+m_{33}\left(-k_{\zeta r 1} \zeta_{r}^{2}-k_{\zeta r 0}\left|\zeta_{r}\right|^{\frac{3}{2}}+\zeta_{r} \Delta \tau_{r}\right)
\end{aligned}
$$

In light of Young's inequality,

$$
\begin{gathered}
\tilde{r} \tilde{d}_{r} \leq \frac{1}{2} \tilde{r}^{2}+\frac{1}{2} \tilde{d}_{r} \\
\tilde{r} \zeta_{r} \leq \frac{1}{2} \tilde{r}^{2}+\frac{1}{2} \zeta_{r}^{2} \\
\zeta_{r} \Delta \tau_{r} \leq \frac{1}{2} \zeta_{r}^{2}+\frac{1}{2} \Delta \tau_{r}^{2} \\
\Theta_{r} \hat{\phi}(r) \tilde{r} \leq c_{r} \Theta_{r} \hat{\phi}^{2}(r) \tilde{r}^{2}+\frac{\Theta_{r}}{4 c_{r}} \\
\tilde{\Theta}_{r} \hat{\Theta}_{r}=\tilde{\Theta}_{r}\left(\Theta_{r}-\tilde{\Theta}_{r}\right) \leq-\frac{\tilde{\Theta}_{r}^{2}}{2}+\frac{\Theta_{r}^{2}}{2}
\end{gathered}
$$

Substituting (72)-(76) into (71), the following equation is obtained:

$$
\begin{aligned}
\dot{V}_{r a} \leq & -k_{\psi} \tilde{\psi}^{2}-U \phi y_{e} \tilde{\psi}-\left(k_{r}-\frac{m_{33} k_{\zeta r}}{2}-1\right) \tilde{r}^{2}-\left(m_{33} k_{\zeta r 1}-\frac{m_{33} k_{\xi_{\zeta r}}}{2}-\frac{m_{33}}{2}\right) \zeta_{r}^{2}+\left(\frac{1}{2}+\frac{m_{33}}{2}\right) \Delta \tau_{r}^{2} \\
& +\frac{1}{2} \tilde{d}_{r}^{2}+m_{33}\left(-\frac{\sigma}{2} \tilde{\Theta}_{r}^{2}+\frac{\sigma}{2} \Theta_{r}^{2}+\frac{\Theta_{r}}{4 c_{r}}\right)
\end{aligned}
$$

Then, $\overline{\tilde{d}}_{r}$ represents the upper limit of yaw rate disturbance estimation.

$$
\begin{aligned}
\dot{V}_{r a} \leq & -k_{\psi} \tilde{\psi}^{2}-U \phi y_{e} \tilde{\psi}-\left(k_{r}-\frac{m_{33} k_{\zeta r}}{2}-1\right) \tilde{r}^{2}-\left(m_{33} k_{\zeta r 1}-\frac{m_{33} k_{\zeta r}}{2}-\frac{m_{33}}{2}\right) \zeta_{r}^{2}+\left(\frac{1}{2}+\frac{m_{33}}{2}\right) \Delta \tau_{r}^{2} \\
& +\frac{1}{2} \tilde{d}_{r}^{2}-\frac{m_{33} \sigma}{2} \tilde{\Theta}_{r}^{2}+m_{33}\left(\frac{\sigma}{2} \Theta_{r}^{2}+\frac{\Theta_{r}}{4 c_{r}}\right)
\end{aligned}
$$


Define

$$
V_{u a}=\frac{m_{11} \tilde{u}^{2}}{2}+\frac{m_{11} \tilde{\Theta}_{\mathrm{u}}^{2}}{2}+\frac{m_{11} \zeta_{u}^{2}}{2}
$$

The time derivative of (79) is

$$
\begin{aligned}
\dot{V}_{u a} & =m_{11} \tilde{u} \dot{\tilde{u}}-m_{11} \tilde{\Theta}_{u} \dot{\hat{\Theta}}_{u}+\mathrm{m}_{11} \zeta_{u} \dot{\zeta}_{u} \\
& =\tilde{u}\left(-k_{u} \tilde{u}+m_{11} \Delta f_{u}-m_{11} c_{u} \hat{\Theta}_{u} \hat{\phi}^{2}(u) \tilde{u}+m_{11} k_{\zeta u} \zeta_{u}+\Delta \tau_{u}+\tilde{d}_{u}\right) \\
& -m_{11} c_{u} \hat{\phi}^{2}(u) \tilde{\Theta}_{u} \tilde{u}^{2}+m_{11} \sigma_{u} \hat{\Theta}_{u} \tilde{\Theta}_{u}+\mathrm{m}_{11}\left(-k_{\zeta u 1} \zeta_{u}^{2}-k_{\zeta u 0}\left|\zeta_{u}\right|^{\frac{3}{2}}+\zeta_{u} \Delta \tau_{u}\right)
\end{aligned}
$$

Substituting (61) into (80),

$$
\begin{aligned}
\dot{V}_{u a} \leq & -k_{u} \tilde{u}^{2}+m_{11} \Theta_{u} \hat{\phi}(u) \tilde{u}-m_{11} c_{u} \hat{\Theta}_{u} \hat{\phi}^{2}(u) \tilde{u}^{2}+m_{11} k_{\zeta u} \zeta_{u} \tilde{u}+\tilde{u} \tilde{d}_{u} \\
& -m_{11} c_{u} \hat{\phi}^{2}(u) \tilde{\Theta}_{u} \tilde{u}^{2}+m_{11} \sigma_{u} \hat{\Theta}_{u} \tilde{\Theta}_{u}+m_{11}\left(-k_{\zeta u 1} \zeta_{u}^{2}-k_{\zeta u 0}\left|\zeta_{u}\right|^{\frac{3}{2}}+\zeta_{u} \Delta \tau_{u}\right)
\end{aligned}
$$

In light of Young's inequality,

$$
\begin{gathered}
\tilde{u} \tilde{d}_{u} \leq \frac{1}{2} \tilde{u}^{2}+\frac{1}{2} \tilde{d}_{u} \\
\tilde{u} \Delta \tau_{u} \leq \frac{1}{2} \tilde{u}^{2}+\frac{1}{2} \Delta \tau_{u}^{2} \\
\zeta_{u} \Delta \tau_{u} \leq \frac{1}{2} \zeta_{u}^{2}+\frac{1}{2} \Delta \tau_{u}^{2} \\
\Theta_{u} \hat{\phi}(u) \tilde{u} \leq c_{u} \Theta_{u} \hat{\phi}^{2}(u) \tilde{u}^{2}+\frac{\Theta_{u}}{4 c_{u}} \\
\tilde{\Theta}_{u} \hat{\Theta}_{u}=\tilde{\Theta}_{u}\left(\Theta_{u}-\tilde{\Theta}_{u}\right) \leq-\frac{\tilde{\Theta}_{u}^{2}}{2}+\frac{\Theta_{u}^{2}}{2}
\end{gathered}
$$

Substituting (82)-(86) into (87), the following equation is obtained:

$$
\begin{aligned}
\dot{V}_{u a} \leq & -\left(k_{u}-\frac{m_{11} k_{\zeta u}}{2}-1\right) \tilde{u}^{2}-\left(\mathrm{m}_{11} k_{\zeta u 1}-\frac{m_{11} k_{\zeta u}}{2}-\frac{\mathrm{m}_{11}}{2}\right) \zeta_{u}^{2}+\left(\frac{\mathrm{m}_{11}}{2}+\frac{1}{2}\right) \Delta \tau_{u}^{2} \\
& +\frac{\tilde{d}_{u}^{2}}{2}+m_{33}\left(-\frac{\sigma_{u}}{2} \tilde{\Theta}_{u}^{2}+\frac{\sigma_{u}}{2} \Theta_{u}^{2}+\frac{\Theta_{u}}{4 c_{u}}\right)
\end{aligned}
$$

Then, $\overline{\tilde{d}}_{u}$ represents the upper limit of surge speed disturbance estimation.

$$
\begin{aligned}
\dot{V}_{u a} \leq & -\left(k_{u}-\frac{m_{11} k_{\zeta u}}{2}-1\right) \tilde{u}^{2}-\left(\mathrm{m}_{11} k_{\zeta u 1}-\frac{m_{11} k_{\zeta u}}{2}-\frac{\mathrm{m}_{11}}{2}\right) \zeta_{u}{ }^{2}+\left(\frac{\mathrm{m}_{11}}{2}+\frac{1}{2}\right) \Delta \tau_{u}{ }^{2} \\
& -\frac{m_{33} \sigma_{u}}{2} \tilde{\Theta}_{u}^{2}+\frac{\tilde{\tilde{d}}_{u}^{2}}{2}+m_{33}\left(\frac{\sigma_{u}}{2} \Theta_{u}^{2}+\frac{\Theta_{u}}{4 c_{u}}\right)
\end{aligned}
$$

Define

$$
V=V_{g}+V_{p}+V_{r a}+V_{u a}
$$


The time derivative of (89) is

$$
\begin{aligned}
\dot{V} \leq & -\left(k_{s}-\frac{1}{2} U\right) x_{e}^{2}-\frac{U}{\sqrt{\Delta^{2}+\left(y_{e}+\Delta \hat{\beta}\right)^{2}}} y_{e}^{2}-\tilde{\beta}^{2}-k_{\psi} \tilde{\psi}^{2} \\
& +|\tilde{\beta}|\left(\frac{C_{\beta}}{k_{\beta}}+\beta^{*}\right)+\frac{1}{2} U \beta^{* 2}-2 D_{1 \min } V_{p}-\left(k_{u}-\frac{m_{11} k_{\zeta u}}{2}-1\right) \tilde{u}^{2} \\
& -\left(\mathrm{m}_{11} k_{\zeta u 1}-\frac{m_{11} k_{\zeta u}}{2}-\frac{\mathrm{m}_{11}}{2}\right) \zeta_{u}^{2}-\left(k_{r}-\frac{m_{33} k_{\zeta r}}{2}-1\right) \tilde{r}^{2}-\left(m_{33} k_{\zeta r 1}-\frac{m_{33} k_{\zeta r}}{2}-\frac{m_{33}}{2}\right) \zeta_{r}^{2} \\
& -\frac{m_{11} \sigma_{u}}{2} \tilde{\Theta}_{u}^{2}-\frac{m_{33} \sigma_{r}}{2} \tilde{\Theta}_{r}^{2}+\left(\frac{1}{2}+\frac{m_{33}}{2}\right) \Delta \tau_{r}^{2}+\left(\frac{\mathrm{m}_{11}}{2}+\frac{1}{2}\right) \Delta \tau_{u}^{2} \\
& +\frac{\tilde{\tilde{d}}_{u}^{2}}{2}+\frac{\tilde{\tilde{d}}_{r}^{2}}{2}+m_{11}\left(\frac{\sigma_{u}}{2} \Theta_{u}^{2}+\frac{\Theta_{u}}{4 c_{u}}\right)+m_{33}\left(\frac{\sigma_{r}}{2} \Theta_{r}^{2}+\frac{\Theta_{r}}{4 c_{r}}\right)
\end{aligned}
$$

Hence,

$$
\dot{V} \leq-2 \mu V+C
$$

where

$$
\begin{aligned}
& \mu=\min \left\{\left(k_{s}-\frac{1}{2} U\right), \frac{U}{\sqrt{\Delta^{2}+\left(y_{e}+\Delta \hat{\beta}\right)^{2}}}, k_{\beta}, k_{\psi}, \frac{1}{m_{33}}\left(k_{r}-\frac{m_{33} k_{\zeta r}}{2}-1\right), \frac{1}{m_{11}}\left(k_{u}-\frac{m_{11} k_{\zeta u}}{2}-1\right),\right. \\
& \left.\left(k_{\zeta r 1}-\frac{k_{\zeta r}}{2}-\frac{1}{2}\right),\left(k_{\zeta u 1}-\frac{k_{\zeta u}}{2}-\frac{1}{2}\right), \frac{\sigma_{u}}{2}, \lambda_{\min }(K)\left(D_{1}\right), \frac{\sigma_{r}}{2}\right\} \\
& C=\frac{\tilde{d}_{u}^{2}}{2}+\frac{\tilde{\tilde{d}}_{r}^{2}}{2}+|\tilde{\beta}|\left(\frac{C_{\beta}}{k_{\beta}}+\beta^{*}\right)+\frac{1}{2} U \beta^{* 2}+\left(\frac{1}{2}+\frac{m_{33}}{2}\right) \Delta \tau_{r}^{2}+\left(\frac{\mathrm{m}_{11}}{2}+\frac{1}{2}\right) \Delta \tau_{u}^{2} \\
& \quad+m_{11}\left(\frac{\sigma}{2} \Theta_{u}^{2}+\frac{\Theta_{u}}{4 c_{u}}\right)+m_{33}\left(\frac{\sigma}{2} \Theta_{r}^{2}+\frac{\Theta_{r}}{4 c_{r}}\right)
\end{aligned}
$$

Based on the estimator (91),

$$
0 \leq V(t) \leq \frac{C}{2 \mu}+\left[V(0)-\frac{C}{2 \mu}\right] e^{-\mu t}
$$

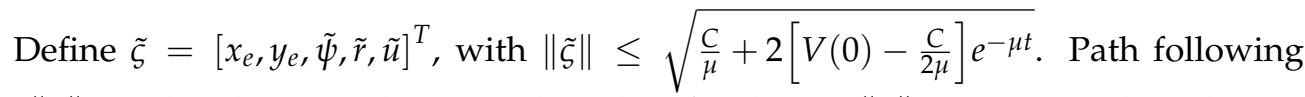
error $\|\tilde{\zeta}\|$ is related to $\mu$ and $C$. For the sake of reducing $\|\tilde{\zeta}\|$, appropriately reduce $\mu$. For a given $\Lambda_{i} \geq \sqrt{\frac{c}{2 \mu}}\left(i=x_{e}, y_{e}, \tilde{\psi}, \tilde{r}, \tilde{u}\right)$, a time constant $T>0$ exists. When $t>T$, $\|\tilde{\zeta}\| \leq \Lambda_{i}\left(i=x_{e}, y_{e}, \tilde{\psi}, \tilde{r}, \tilde{u}\right)$. The path following error of an underactuated USV converges to a compact set $\Omega=\left\{\|\tilde{\zeta}\| \leq \sqrt{\frac{C}{2 \mu}}\right\}$. By measuring reasonable parameters, the path following error of an underactuated USV can converge to any small neighborhood near zero.

\section{Numerical Simulations}

For the sake of testifying to the validity of the proposed control policy, simulation research is implemented. The research objective refers to Paper [39] for revealing the nominal physical parameters. On this basis, to testify to the superiority of the adaptive finite-time control scheme (AFTC) of this paper, the simulation of straight-line and curve path tracking is implemented under strong and weak interference and compared with the adaptive neural network controller (ANNC) designed in Paper [17]. 


\subsection{Path Following under Weak Interference}

\subsubsection{Straight-Line Path Following under Weak Interference}

The design parameters are determined as follows: $u_{d}=1 \mathrm{~m} / \mathrm{s}, k_{\beta}=6, \Delta=10$, $k_{s}=10, k_{r}=10, k_{\psi}=12, c_{r}=0.83, \sigma_{r}=0.001, k_{\zeta r}=0.01, k_{\zeta r 1}=1, k_{\zeta r 0}=0.01$, $k_{u}=25, c_{u}=0.95, \sigma_{u}=0.001, k_{\zeta u}=0.01, k_{\zeta u 1}=1, k_{\zeta u 0}=0.01, \gamma=0.6, D_{1}=$ $\operatorname{diag}\{2,2,2\}, D_{2}=\operatorname{diag}\{1,1,1\}, \tau_{u \max }=15(\mathrm{~N}), \tau_{u \min }=-15(\mathrm{~N}), \tau_{r \max }=10(\mathrm{~N})$, $\tau_{r \min }=-10(\mathrm{~N}), \iota_{1}=100, a_{1}=1, a_{2}=1.2, a_{3}=2, \iota_{2}=80, a_{4}=1$ and $a_{5}=2$. $[x(0), y(0), \psi(0), u(0), v(0), r(0)]=[0,5,-\pi / 18,0.2,0,0]$ denotes the initial state of the USV. $p_{d}(\theta)=[\theta, \theta]^{T}$ denotes a desired straight-line geometrical path. Moreover, in light of [16], time-dependent disturbances are assumed as

$$
\left\{\begin{array}{l}
b_{u}=2 \sin (0.1 t)+1 \cos (0.05 t) \\
b_{v}=1 \sin (0.05 t) \\
b_{r}=2 \sin (0.1 t)+0.2 \cos (0.05 t)
\end{array}\right.
$$

The straight-line path following simulation under weak interference is plotted in Figure 4.

As revealed in Table 1, considering $x_{e}$ and $y_{e}$, the control policy proposed in this paper has notable performance. Figure $4 \mathrm{a}$ plots that the reference straight-line can be tracked precisely by the USV. Meanwhile, we can observe that the USV of scheme AFTC converges to the desired path within a short time and has smaller overshoot than scheme ANNC. It can be observed from Figure $4 \mathrm{~b}$ that the along-track error scheme of AFTC converges faster than that of scheme ANNC by nearly 8 seconds; the cross-track error of scheme AFTC converges nearly 10 seconds faster than that of scheme ANNC, and the overshoot is $30 \%$ smaller. The along-track error and cross-track error of the USV of scheme AFTC can converge to zero precisely within a short time and have smaller overshoot than scheme ANNC. Figure $4 \mathrm{c}$ illustrates that heading and surge velocity errors converge quickly in a short time. The surge velocity error of scheme AFTC converges faster than that of scheme ANNC by nearly 7 seconds and the heading velocity error of scheme AFTC converges faster than that of scheme ANNC by nearly 16 seconds. Scheme AFTC converges faster than scheme ANNC. Figure $4 \mathrm{~d}$ shows estimations of disturbances. We can see that the disturbance observers perfectly estimate external disturbances in the initial phase of control. From Figure $4 \mathrm{e}$, we can also observe the control inputs of $\tau_{u}$ and $\tau_{r}$. Due to error, control gain, the algorithm itself and other factors, the control input has a large value in the initial stage of control. From a practical point of view, it is essential to take the input saturation into account in the design of the proposed strategy. Figure $4 \mathrm{f}$ shows that with estimations $\hat{\Phi}_{u}$ and $\hat{\Phi}_{r}$, under weak interference, the model has about 10 percent deviation. Because the deviation of the model is compensated, it brings better control effect to the controller. Figure $4 \mathrm{~g}$ demonstrates that the sideslip angle can be evaluated quickly in the presence of a disturbance.

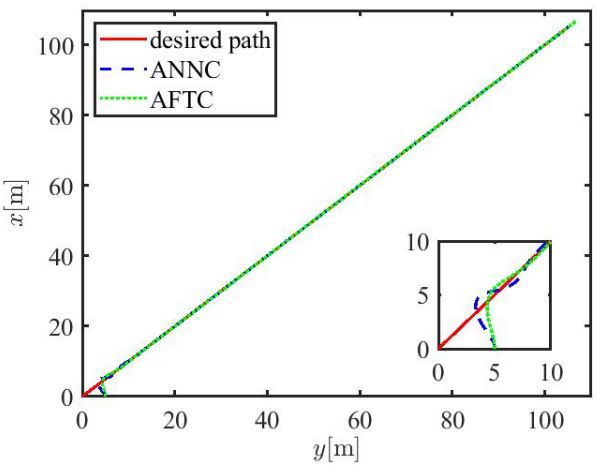

(a)
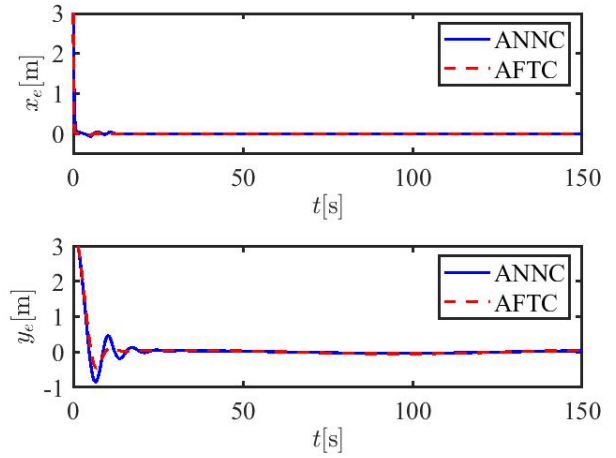

(b)

Figure 4. Cont. 

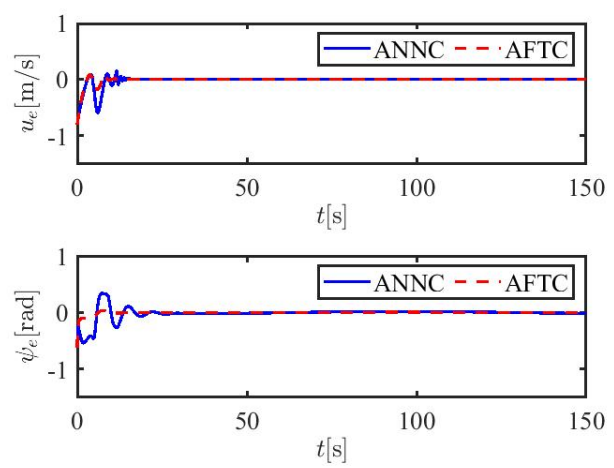

(c)
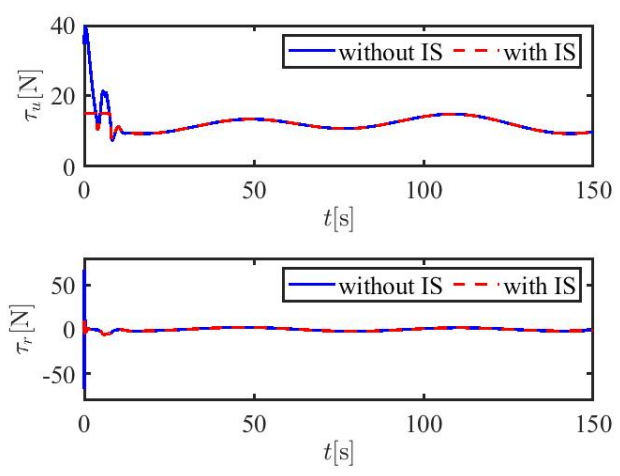

(e)

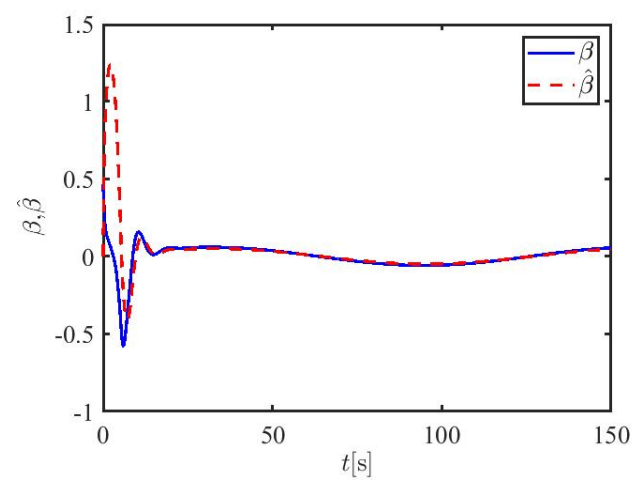

(g)
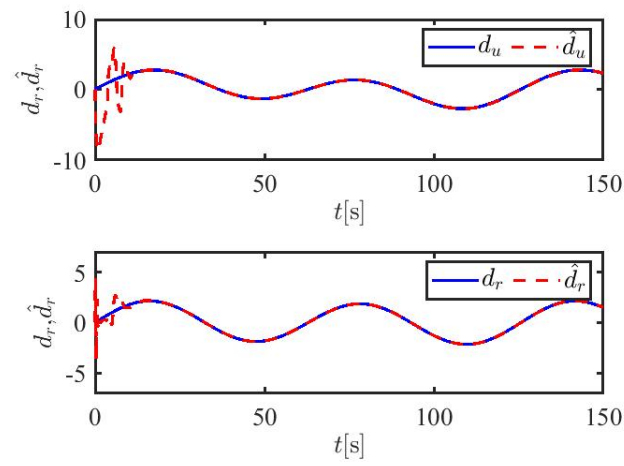

(d)
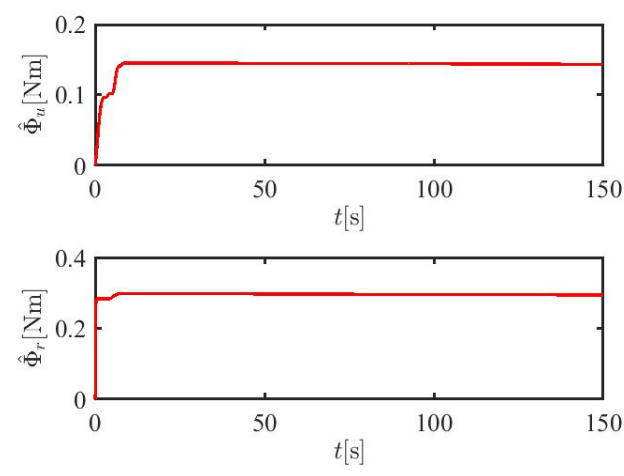

(f)

Figure 4. The results of the straight-line path following under weak interference: (a) Path following result. (b) Along-track and cross-track error. (c) Profile of the heading and surge velocity tracking. (d) Estimations of disturbance. (e) Profile of the control inputs. (f) Estimations $\hat{\Phi}_{r}$ and $\hat{\Phi}_{r}$. (g) Estimations of sideslip angle.

Table 1. Performance indicator straight-line path following under weak interference.

\begin{tabular}{ccc}
\hline Performance Indicator & AFTC & ANNC \\
\hline $\operatorname{IEA}\left(x_{e}\right)$ & 0.36 & 0.848 \\
$\operatorname{IEA}\left(y_{e}\right)$ & 8.74 & 10.2 \\
\hline
\end{tabular}




\subsubsection{Curve Path Following under Weak Interference}

Other design parameters remain unchanged except for $c_{r}=8.3$. $p_{d}(\theta)=$ $[20 \sin (\theta / 20)+\theta, \theta]^{T}$ denotes a desired curve geometrical path. The simulation research of curve path following is plotted in Figure 5.

The simulation research of curve path following under weak interference is plotted in Figure 5.

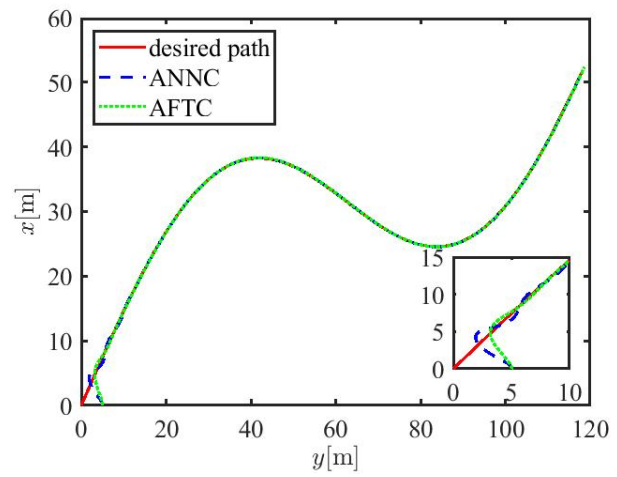

(a)
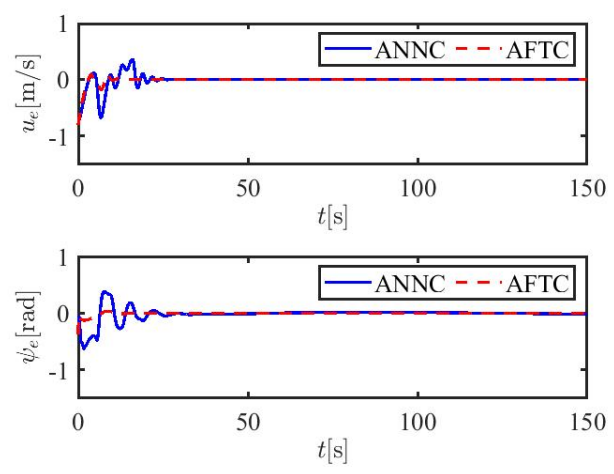

(c)
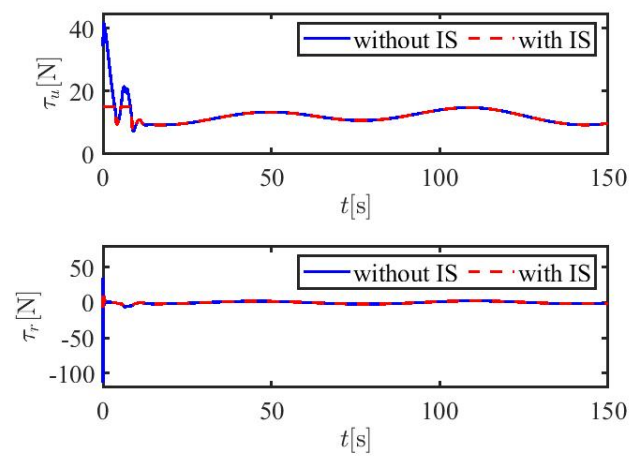

(e)
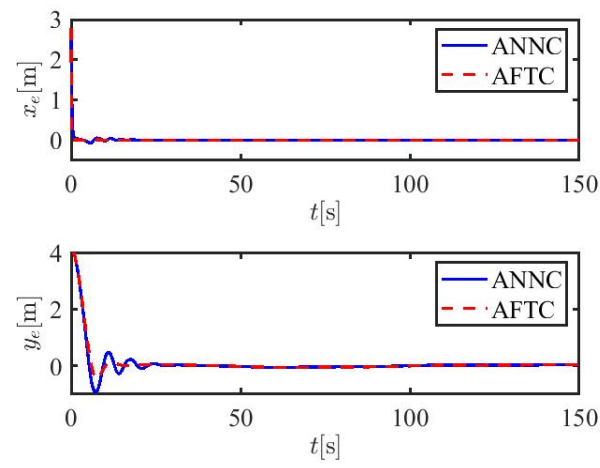

(b)
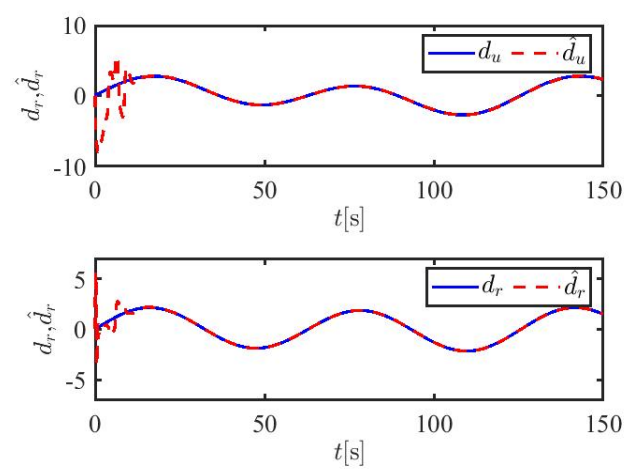

(d)
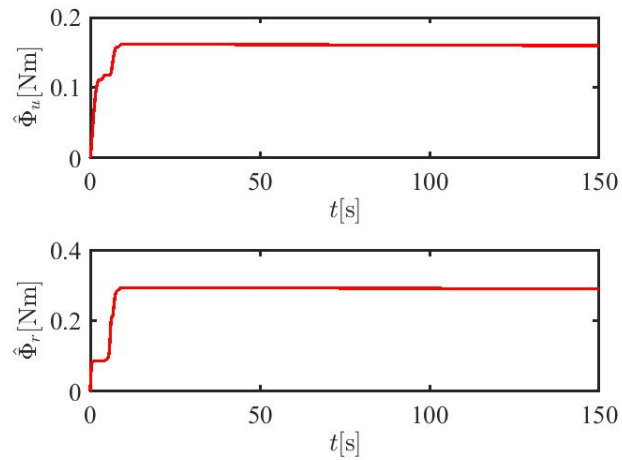

(f)

Figure 5. Cont. 


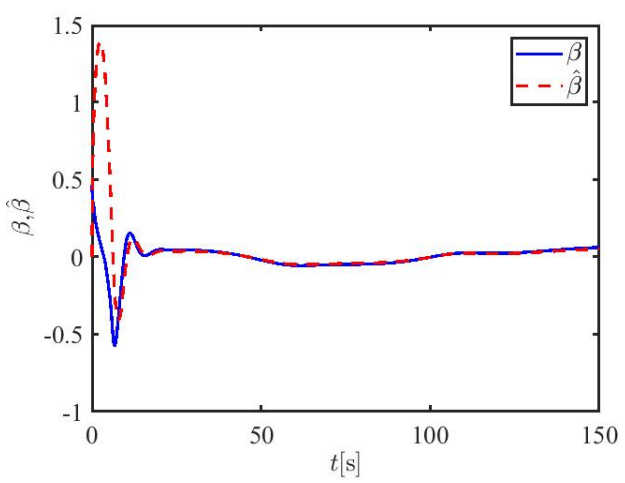

$(\mathrm{g})$

Figure 5. The results of the curve path following under weak interference: (a) Path following result. (b) Along-track and cross-track error. (c) Profile of the heading and surge velocity tracking. (d) Estimations of disturbance. (e) Profile of the control inputs. (f) Estimations $\hat{\Phi}_{r}$ and $\hat{\Phi}_{r}$. (g) Estimations of sideslip angle.

As revealed in Table 2, considering $x_{e}$ and $y_{e}$, the control policy proposed in this paper has notable performance advantages. Figure 5 a plots that the assignment of path following can still be put into effect well with only one parameter modified. It is indicated that the proposed path following strategy has excellent adaptability. The along-track error and cross-track error of the USV are revealed in Figure 5b. The along-track error of scheme AFTC converges faster than that of scheme ANNC by nearly 10 seconds, the cross-track error of scheme AFTC converges nearly 12 seconds faster than that of scheme ANNC, and the overshoot is $33 \%$ smaller. In addition, it still can retain sterling control performance. The heading and surge velocity errors, which are depicted in Figure $5 c$, resemble straightline path following. The surge velocity error of scheme AFTC converges faster than that of scheme ANNC by nearly 8 seconds and the heading velocity error of scheme AFTC converges faster than that of scheme ANNC by nearly 18 seconds. The estimations of disturbances can be observed in Figure $5 \mathrm{~d}$. Similarly, the control inputs $\tau_{u}$ and $\tau_{r}$ are depicted in Figure 5e. Estimations $\hat{\Phi}_{u}$ and $\hat{\Phi}_{r}$ are displayed in Figure 5f. Estimations of sideslip angle are displayed in Figure 5g.

Table 2. Performance indicator of curve path following under weak interference.

\begin{tabular}{ccc}
\hline Performance Indicator & AFTC & ANNC \\
\hline IEA $x_{e}$ & 0.29 & 0.719 \\
IEA $y_{e}$ & 12.1 & 13.8 \\
\hline
\end{tabular}

\subsection{Following under Strong Interference}

\subsubsection{Straight-Line Path Following under Strong Interference}

The design parameters are determined as follows: $u_{d}=1 \mathrm{~m} / \mathrm{s}, k_{\beta}=6, \Delta=10$, $k_{s}=10, \mathrm{k}_{\mathrm{r}}=12, k_{\psi}=12, c_{r}=1.5, \sigma_{r}=0.001, k_{\zeta r}=0.01, k_{\zeta r 1}=1, k_{\zeta r 0}=0.01$, $k_{u}=30, c_{u}=1.9, \sigma_{u}=0.001, k_{\zeta u}=0.01, k_{\zeta u 1}=1, k_{\zeta u 0}=0.01, \gamma=0.6, D_{1}=$ $\operatorname{diag}\{2,2,2\}, D_{2}=\operatorname{diag}\{1,1,1\}, \tau_{u \max }=16(\mathrm{~N}), \tau_{u \min }=-16(\mathrm{~N}), \tau_{r \max }=10(\mathrm{~N})$, $\tau_{r \min }=-10(\mathrm{~N}), \iota_{1}=100, a_{1}=1, a_{2}=1.2, a_{3}=2, \iota_{2}=80, a_{4}=1$ and $a_{5}=2$. $[x(0), y(0), \psi(0), u(0), v(0), r(0)]=[0,5,-\pi / 18,0.2,0,0]$ denotes the initial state of the USV. $p_{d}(\theta)=[\theta, \theta]^{\mathrm{T}}$ denotes a desired straight-line geometrical path. Moreover, 
considering the actual situation of strong interference, combined with [16], time-dependent disturbances are assumed as

$$
\left\{\begin{array}{l}
b_{u}=3 \sin (0.1 t)+1.5 \cos (0.05 t) \\
b_{v}=2 \sin (0.05 t) \\
b_{r}=2 \sin (0.1 t)+3 \cos (0.05 t)
\end{array}\right.
$$

As revealed in Table 3, AFTC has notable performance. The simulation research of straight-line path following under strong interference is plotted in Figure 6a-g. Figure $6 \mathrm{f}$ shows that for estimations $\hat{\Phi}_{u}$ and $\hat{\Phi}_{r}$, under weak interference, the model has about $20 \%$ deviation. Under strong interference, the model parameters are effectively compensated, which has practical significance. The overall control effect resembles straight-line path following under weak interference.

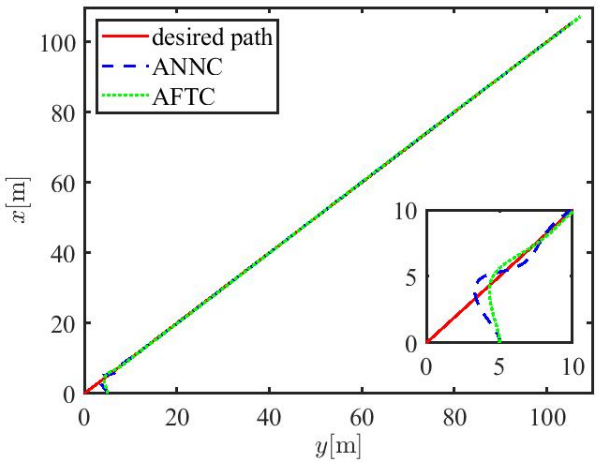

(a)
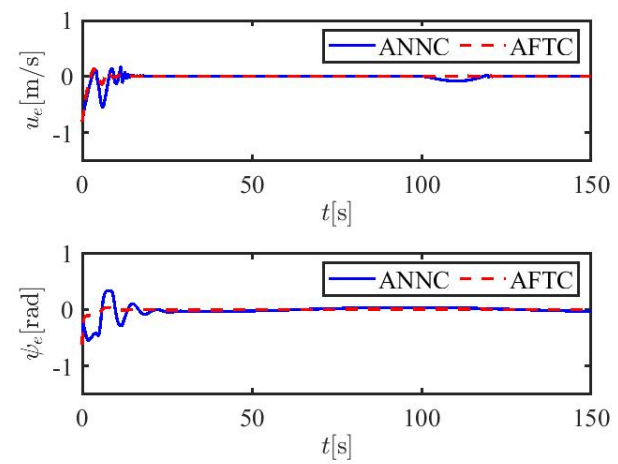

(c)
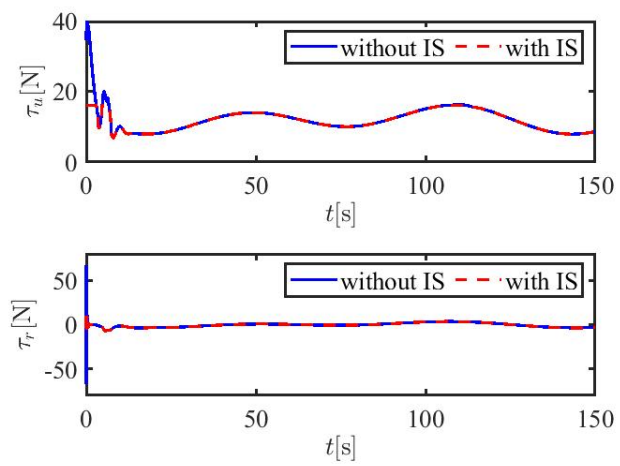

(e)
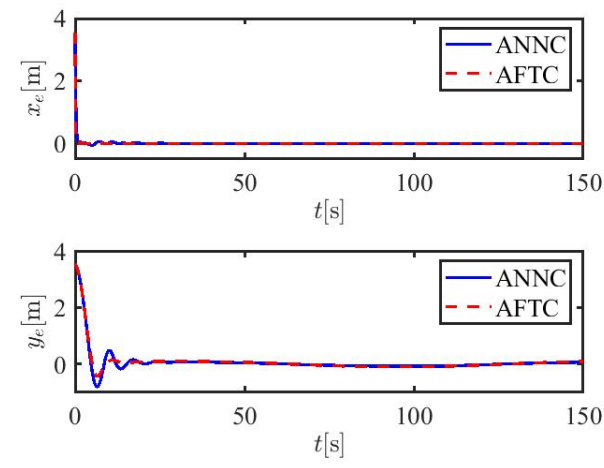

(b)
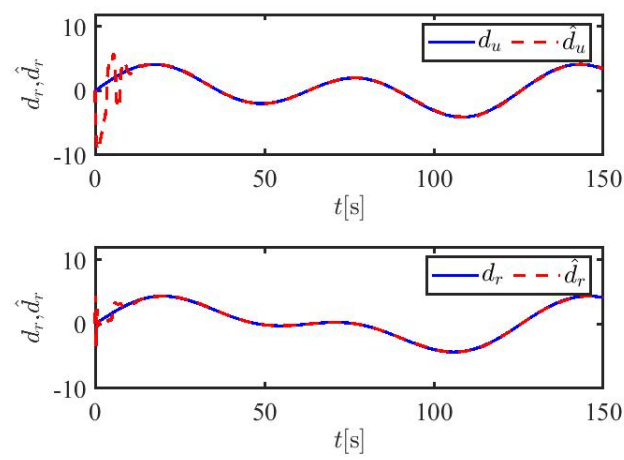

(d)
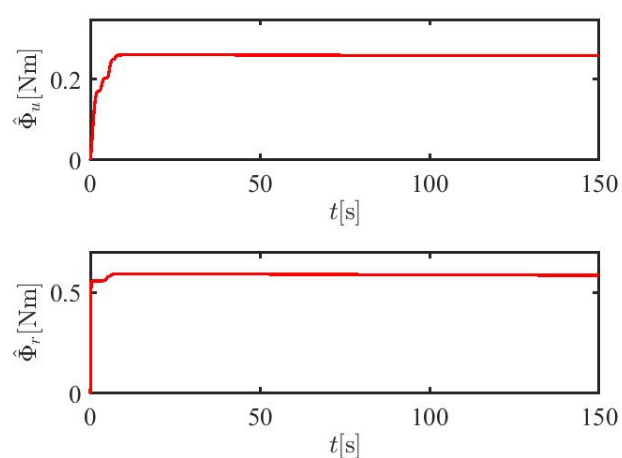

(f)

Figure 6. Cont. 


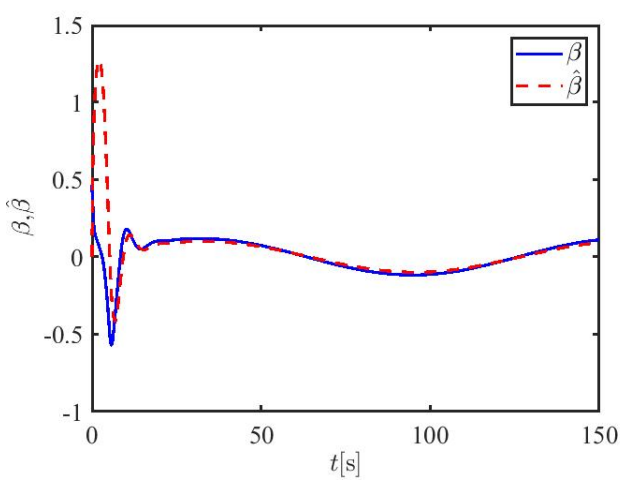

(g)

Figure 6. The results of the straight-line path following: (a) Path following result. (b) Along-track and cross-track error. (c) Profile of the heading and surge velocity tracking. (d) Estimations of disturbance. (e) Profile of the control inputs. (f) Estimations $\hat{\Phi}_{r}$ and $\hat{\Phi}_{r}$. (g) Estimations of sideslip angle.

Table 3. Performance indicator of straight-line path following under strong interference.

\begin{tabular}{ccc}
\hline Performance Indicator & AFTC & ANNC \\
\hline IEA $x_{e}$ & 0.450 & 0.926 \\
IEA $y_{e}$ & 9.17 & 13.3 \\
\hline
\end{tabular}

\subsubsection{Curve Path Following under Strong Interference}

Other design parameters remain unchanged except for $c_{r}=7.6$ and $c_{u}=1.8$. In addition, $p_{d}(\theta)=[20 \sin (\theta / 20)+\theta, \theta]^{T}$ denotes the desired curve geometrical path.

The simulation research of curve path following under strong interference is plotted in Figure 7.

As revealed in Table 4, considering $x_{e}$ and $y_{e}$, the control policy proposed in this paper has notable performance advantages. The simulation research of curve path following under strong interference is plotted in Figure $7 \mathrm{a}-\mathrm{g}$. Under strong interference, Figure $7 \mathrm{f}$ shows that for estimations $\hat{\Phi}_{u}, \hat{\Phi}_{r}$, the model has about $20 \%$ deviation. Under strong interference, the model deviation parameters are effectively compensated, which brings good control effect to curve path following. The overall control effect is similar to that of curve path following under weak interference. Consequently, the numerical simulations of straight-line path geometrical following and curve geometrical path following have realized excellent results, testifying to the validity and superiority of the IAILOS guidance scheme and control scheme proposed in this paper.

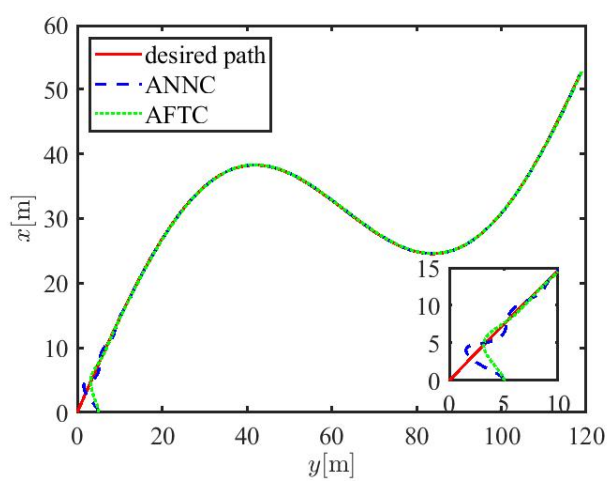

(a)
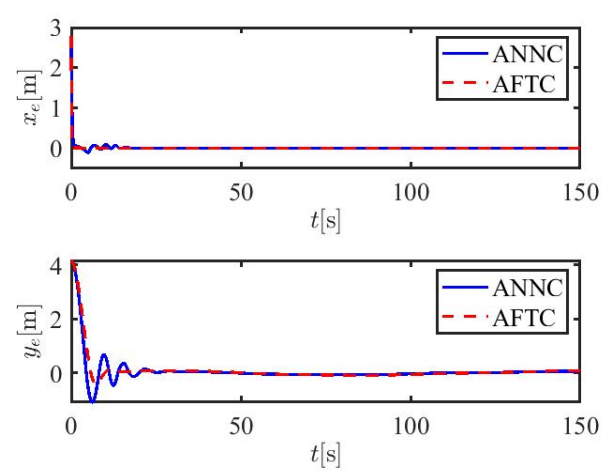

(b)

Figure 7. Cont. 

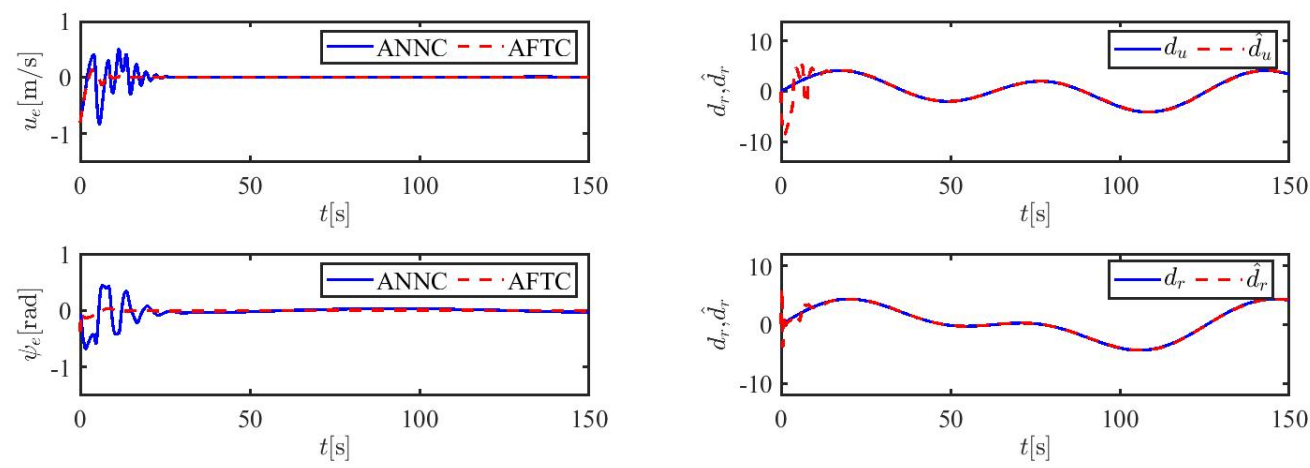

(c)

(d)
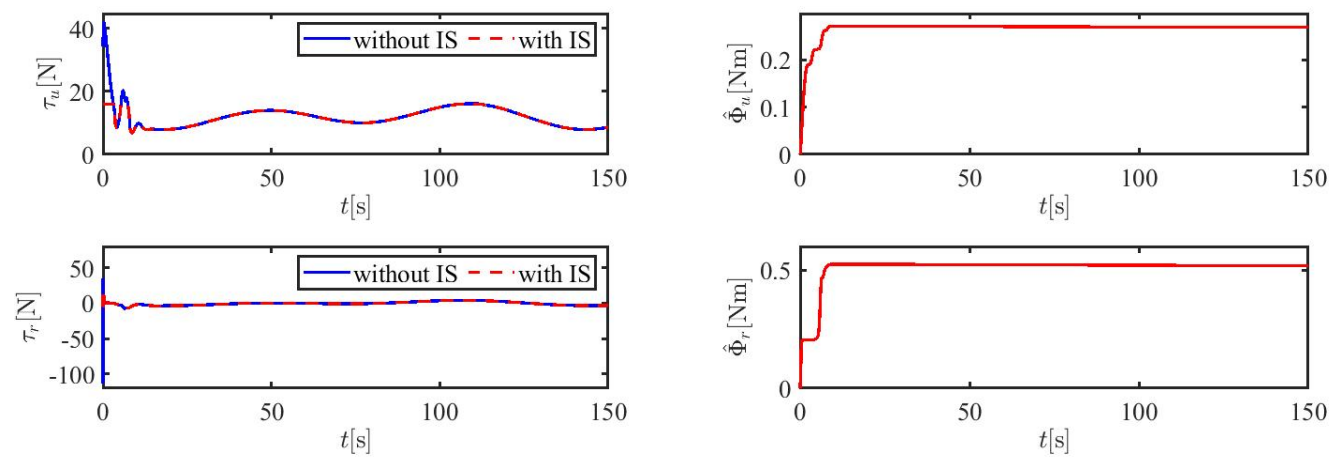

(e)

(f)

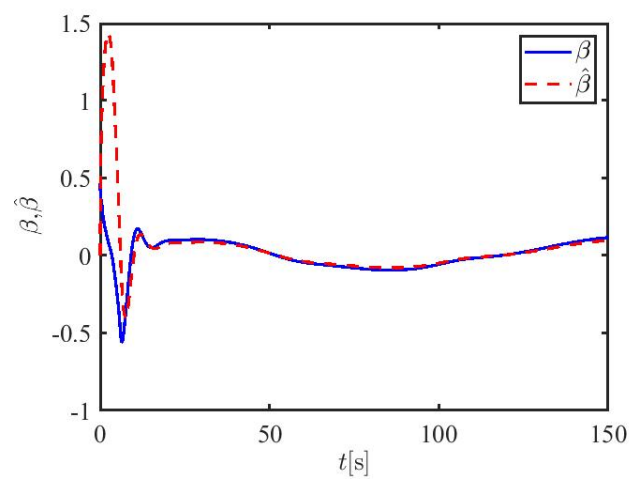

(g)

Figure 7. The results of the curve path following: (a) Path following result. (b) Along-track and cross-track error. (c) Profile of the heading and surge velocity tracking. (d) Estimations of disturbance. (e) Profile of the control inputs. (f) Estimations $\hat{\Phi}_{r}$ and $\hat{\Phi}_{r}$. (g) Estimations of sideslip angle.

Table 4. Performance indicator of curve path following under weak interference.

\begin{tabular}{ccc}
\hline Performance Indicator & AFTC & ANNC \\
\hline $\operatorname{IEA}\left(x_{e}\right)$ & 0.36 & 0.848 \\
$\operatorname{IEA}\left(y_{e}\right)$ & 8.74 & 10.2 \\
\hline
\end{tabular}

\section{Conclusions}

In this paper, a series of schemes for underactuated USV path following is proposed. Initially, the guidance strategy of IALOS is introduced to evaluate the time-varying sideslip angle. Second, a USV path following controller is designed subject to external time-varying 
disturbance, deviation of vessel model parameters and actuator saturation. The finite-time disturbance observer is introduced to evaluate the external disturbance quickly, the singleparameter neural network is used to eliminate the model error and the finite-time auxiliary dynamic system is presented to accelerate the convergence speed. The introduction of TDS improves the problem of "differential explosion". In addition, the stability of the closed-loop system is demonstrated by using Lyapunov functions. Finally, the validity of the path following strategy is demonstrated by four numerical simulations.

Despite that this paper takes as many practical circumstances into consideration as possible, there still exist some issues to be settled. Due to the introduction of too many design parameters, it may bring some inconvenience into the process of parameter adjustment. Ocean currents are not considered in this paper. There is no constraint on the position error in the procedure of path following. These problems will be solved in the next work.

Author Contributions: The work introduced here is completed with the cooperation of all authors. Y.F. conducted the full text and visualized the framework. X.Z. designed and composed the paper. G.W. visualized the idea. D.M. analyzed the simulations. The manuscript was contributed to and given approval by all authors. All authors have read and agreed to the published version of the manuscript.

Funding: This work was supported in part by the National Natural Science Foundation of China under Grant 51609033, the Natural Science Foundation of Liaoning Province under Grant 20180520005, the Key Development Guidance Program of Liaoning Province of China under Grant 2019JH8/10100100, the Soft Science Research Program of Dalian City of China under Grant 2019J11CY 014 and Fundamental Research Funds for the Central Universities under Grants 3132021106, 3132019005 and 3132019311.

Institutional Review Board Statement: Not applicable.

Informed Consent Statement: Not applicable.

Data Availability Statement: Not applicable.

Conflicts of Interest: The authors announce no conflict of interest.

\begin{tabular}{|c|c|}
\hline \multicolumn{2}{|c|}{ Abbreviations } \\
\hline The fo & ing abbreviations are used in this manus \\
\hline USV & unmanned surface vehicle \\
\hline LOS & line-of-sight \\
\hline ALOS & adaptive line-of-sight \\
\hline PLOS & predictor-based line-of-sight \\
\hline IALOS & improved adaptive integral line-of-sight \\
\hline SMC & sliding mode control \\
\hline TDS & the higher-order tracking differentiator \\
\hline DSC & dynamic surface control \\
\hline MPC & model predictive control \\
\hline MLP & minimal learning parameter \\
\hline FCDO & finite-time disturbance observer \\
\hline ANNC & adaptive neural network control \\
\hline AFTC & adaptive finite-time control \\
\hline
\end{tabular}

\section{References}

1. Mu, D.; Wang, G.; Fan, Y.; Qiu, B.; Sun, X. Adaptive Course Control based on Trajectory Control for Unmanned surface Vehicle with Unmodeled Dynamics and Input Saturation. Neurocomputing 2018, 303, 1-10. [CrossRef]

2. Park, B.S.; Yoo, S.J. Robust fault-tolerant tracking with predefined performance for underactuated surface vessels. Ocean Eng. 2016, 115, 159-167. [CrossRef]

3. Wang, N.; Er, M.J.; Sun, J.C.; Liu, Y.C. Adaptive Robust Online Constructive Fuzzy Control of a Complex Surface Vehicle System. IEEE Trans. Cybern. 2015, 46, 1511-1523. [CrossRef]

4. Lu, Y.; Zhang, G.; Qiao, L.; Zhang, W. adaptive output-feedback formation control for underactuated surface vessels. Int. J. Control 2020, 93, 400-409. [CrossRef] 
5. Qiu, B.; Wang, G.; Fan, Y. Predictor LOS-based trajectory linearization control for path following of underactuated unmanned surface vehicle with input saturation. Ocean Eng. 2020, 214, 107874. [CrossRef]

6. Miao, J.; Wang, S.; Tomovic, M.M.; Zhao, Z. Compound line-of-sight nonlinear path following control of underactuated marine vehicles exposed to wind, waves, and ocean currents. Nonlinear Dyn. 2017, 89, 2441-2459. [CrossRef]

7. Mu, D.; Wang, G.; Fan, Y.; Bai, Y.; Zhao, Y. Path following for podded propulsion unmanned surface vehicle: Theory, simulation and experiment. IEEJ Trans. Electr. Electron. Eng. 2018, 13, 911-923. [CrossRef]

8. Fossen, T.I.; Pettersen, K.Y. On uniform semiglobal exponential stability (usges) of proportional line-of-sight guidance laws. Automatica 2014, 50, 2912-2917. [CrossRef]

9. Borhaug, E.; Pavlov, A.; Pettersen, K.Y. Integral LOS control for path following of underactuated marine surface vessels in the presence of constant ocean currents. In Proceedings of the 2008 47th IEEE Conference on Decision and Control, Cancun, Mexico, 9-11 December 2008.

10. Lekkas, A.M.; Fossen, T.I. Integral LOS Path Following for Curved Paths Based on a Monotone Cubic Hermite Spline Parametrization. IEEE Trans. Control Syst. Technol. 2014, 22, 2287-2301. [CrossRef]

11. Caharija, W.; Pettersen, K.Y.; Bibuli, M.; Calado, P.; Zereik, E.; Braga, J.; Gravdahl, J.T.; Sørensen, A.J.; Milovanović, M.; Bruzzone, G. Integral Line-of-Sight Guidance and Control of Underactuated Marine Vehicles: Theory, Simulations, and Experiments. IEEE Trans. Control Syst. Technol. 2016, 24, 1623-1642. [CrossRef]

12. Fossen, T.I.; Pettersen, K.Y.; Galeazzi, R. Line-of-Sight Path Following for Dubins Paths with Adaptive Sideslip Compensation of Drift Forces. IEEE Trans. Control Syst. Technol. 2015, 23, 820-827. [CrossRef]

13. Zheng, Z.; Sun, L. Path following control for marine surface vessel with uncertainties and input saturation. Neurocomputing 2016, 177, 158-167. [CrossRef]

14. Liu, L.; Wang, D.; Peng, Z.; Wang, H. Predictor-based LOS guidance law for path following of underactuated marine surface vehicles with sideslip compensation. Ocean Eng. 2016, 124, 340-348. [CrossRef]

15. Liu, L.; Wang, D.; Peng, Z. ESO-Based Line-of-Sight Guidance Law for Path Following of Underactuated Marine Surface Vehicles With Exact Sideslip Compensation. IEEE J. Ocean. Eng. 2016, 42, 477-487. [CrossRef]

16. Nie, J.; Lin, X. Improved Adaptive integral Line-of-Sight Guidance Law and Nonlinear Adaptive Fuzzy Path Following control for Underactuated MSV. ISA Trans. 2019, 94, 151-163. [CrossRef]

17. Fan, Y.; Huang, H.; Tan, Y. Robust Adaptive Path Following Control of an Unmanned Surface Vessel Subject to Input Saturation and Uncertainties. Appl. Sci. 2019, 9, 1815. [CrossRef]

18. Xia, R.; Chen, M.; Wu, Q.; Wang, Y. Neural network based integral sliding mode optimal flight control of near space hypersonic vehicle. Neurocomputing 2019, 379, 41-52. [CrossRef]

19. Do, K.D. Global robust adaptive path-tracking control of underactuated ships under stochastic disturbances. Ocean. Eng. 2016, 111, 267-278. [CrossRef]

20. Ashrafiuon, H.; Muske, K.R.; McNinch, L.C.; Soltan, R.A. Sliding-Mode Tracking Control of Surface Vessels. IEEE Trans. Ind. Electron. 2008, 55, 4004-4012. [CrossRef]

21. Fan, Y.; Liu, B.; Wang, G.; Mu, D. Adaptive Fast Non-Singular Terminal Sliding Mode Path Following Control for an Underactuated Unmanned Surface Vehicle with Uncertainties and Unknown Disturbances. Sensors 2021, 21, 7454. [CrossRef]

22. Zheng, Z.; Feroskhan, M. Path Following of a Surface Vessel with Prescribed Performance in the Presence of Input Saturation and External Disturbances. IEEE/ASME Trans. Mechatron. 2017, 22, 2564-2575. [CrossRef]

23. Mu, D.D.; Wang, G.F.; Fan, Y.S. Tracking control of Podded Propulsion Unmanned Surface Vehicle with Unknow Dynamics and Disturbance Under Input Saturation. Int. J. Control Autom. Syst. 2018, 16, 1905-1915. [CrossRef]

24. Fu, M.; Wang, L. Disturbance Observer Based Path Following Control of Unmanned Surface Vessel with Time-varying Current. In Proceedings of the 38th Chinese Control Conference, Guangzhou, China, 27-30 July 2019.

25. Lu, Y.; Zhang, G.; Qiao, L.; Zhang, W. Robust adaptive formation control for underactuated surface autonomous vessels based on MLP and DOB. Nonlinear Dyn. 2018, 94, 503-519. [CrossRef]

26. Zhang, G.; Zhang, X. Concise robust adaptive path following control of underactuated ships using DSC and MLP. IEEE J. Ocean. Eng. 2014, 39, 684-694. [CrossRef]

27. Dai, S.; Wang, M.; Wang, C. Neural learning control of marine surface vessels with guaranteed transient tracking performance. IEEE Trans. Ind. Electron. 2016, 63, 1717-1727. [CrossRef]

28. Pan, C.Z.; Lai, X.Z.; Yang, S.X.; Wu, M. An efficient neural network aprroach to tracking control of an autonomous surface vehicle with unknown dynamics. Expert Syst. Appl. 2013, 40, 1629-1635. [CrossRef]

29. Zhu, G.; Ma, Y.; Hu, S. Single-parameter-learning-based finite-time tracking control of underactuated MSVs under input saturation Control Eng. Pract. 2020, 105, 104652. [CrossRef]

30. Wischnewski, A.; Betz, J.; Lohmann, B. Real-Time Learning of Non-Gaussian Uncertainty Models for Autonomous Racing. In Proceedings of the 2020 59th IEEE Conference on Decision and Control (CDC), Jeju, Korea, 14-18 December 2020; pp. 609-615. [CrossRef]

31. Wischnewski, A.; Betz, J.; Lohmann, B. A Model-Free Algorithm to Safely Approach the Handling Limit of an Autonomous Racecar. In Proceedings of the 2019 IEEE International Conference on Connected Vehicles and Expo (ICCVE), Graz, Austria, 4-8 November 2019. [CrossRef] 
32. Rosolia, U.; Carvalho, A.; Borrelli, F. Autonomous racing using learning model predictive control American Control. In Proceedings of the 2017 American Control Conference (ACC), Seattle, WA, USA, 24-26 May 2017; pp. 5115-5120.

33. Rosolia, U.; Borrelli, F. Learning Model Predictive Control for Iterative Tasks: A Computationally Efficient Approach for Linear System. IFAC-PapersOnLine 2017, 50, 3142-3147. [CrossRef]

34. Borrelli, F.; Falcone, P.; Keviczky, T.; Asgari, J.; Hrovat, D. MPC-based approach to active steering for autonomous vehicle systems. Int. J. Veh. Auto Syst. 2005, 3, 265-291. [CrossRef]

35. Fossen, T.I. Marine Control Systems: Guidance, Navigation and Control of Ships, Rigs and Underwater Vehicles. In Marine Cybernetics; Springer: Trondheim, Norway, 2002.

36. Li, J.-H.; Lee, P.-M.; Jun, B.-H.; Lim, Y.-K. Point-to-point navigation of underactuated ships. Automatica 2008, 44, 3201-3205. [CrossRef]

37. Du, H.; Qian, C.; Frye, M.T.; Li, S. Global finite-time stabilisationusing bounded feedback for a class of nonlinear systems. IET Control Theory Appl. 2012, 6, 2326-2336. [CrossRef]

38. Liang, K.; Lin, X.; Chen, Y.; Li, J.; Ding, F. Adaptive sliding mode output feedback control for dynamic positioning ships with input saturation. Ocean Eng. 2020, 206, 107245. [CrossRef]

39. Skjetne, R.; Fossen, T.I.; Kokotović, P.V. Adaptive maneuvering, with experiments, for a model ship in a marine control laboratory. Automatica 2005, 41, 289-298. [CrossRef] 